\title{
Comparison of the Measurement Uncertainties and Errors for the Waveform State Levels Estimated Using the Histogram Mode and Shorth Methods
}

\author{
Nicholas G. Paulter, Jr
}




\section{Comparison of the Measurement Uncertainties and Errors for the Waveform State Levels Estimated Using the Histogram Mode and Shorth Methods}

Nicholas G. Paulter, Jr. Materials Measurement Science Division Material Measurement Laboratory

This publication is available free of charge from:

https://doi.org/10.6028/NIST.TN.2036

February 2019

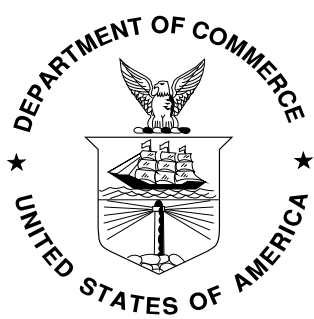

U.S. Department of Commerce Wilbur L. Ross, Jr., Secretary

National Institute of Standards and Technology Walter Copan, NIST Director and Undersecretary of Commerce for Standards and Technology 
Certain commercial entities, equipment, or materials may be identified in this document in order to describe an experimental procedure or concept adequately. Such identification is not intended to imply recommendation or endorsement by the National Institute of Standards and Technology, nor is it intended to imply that the entities, materials, or equipment are necessarily the best available for the purpose.

National Institute of Standards and Technology Technical Note 2036 Natl. Inst. Stand. Technol. Tech. Note 2036, 39 pages (February 2019) CODEN: NTNOEF

This publication is available free of charge from: https://doi.org/10.6028/NIST.TN.2036 


\begin{abstract}
State levels are a fundamental waveform parameter that are essential for the computation of most other waveform parameters. The histogram-mode method has been the mainstay for the estimation of state level and the shorth method was introduced as a potentially more accurate estimator. A comparison of the errors and measurement uncertainties in the values of state level that are computed using the histogram-mode method and the shorth method are presented. The effects of noise and aberrations in this comparison are included. The performance of these methods depends on the types and magnitude of the noise and aberrations.
\end{abstract}

Key words: documentary standards; histogram; measurement uncertainty; mode; shorth; state; state level; waveform; waveform parameter; uncertainty. 


\section{Table of Contents}

1. Background ....................................................................................................................... 1

1.1. Methods for determining level(s) ................................................................. 2

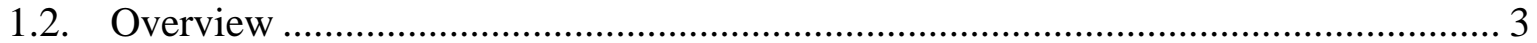

2. Error and measurement uncertainty algorithms ................................................................. 4

2.1. Mode method............................................................................................... 5

2.3. Shorth method ............................................................................................... 7

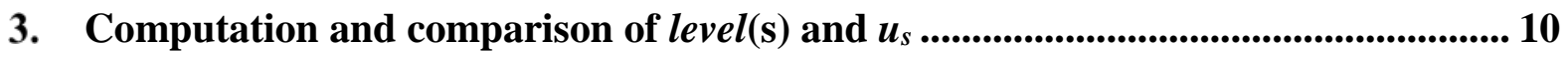

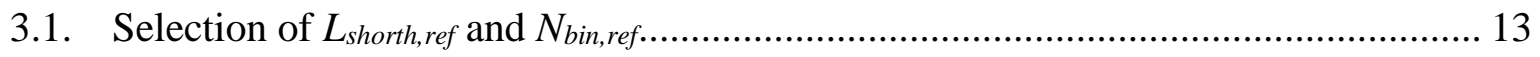

3.2. Comparison of histogram-mode and shorth methods for noisy waveforms with no

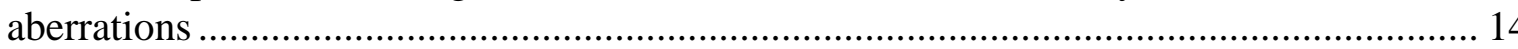

3.3. Comparison of histogram-mode and shorth methods for noisy waveforms with

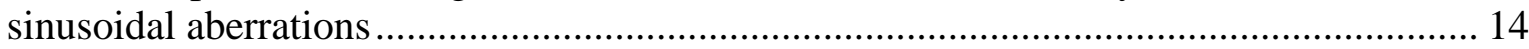

3.4. Comparison of histogram-mode and shorth methods for noisy waveforms with rectangular-pulse-train aberrations .................................................................................... 19

3.5. Histogram-mode and shorth methods for noisy waveforms with impulse-like

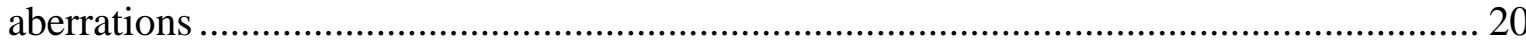

3.6. Collective comparison of histogram-mode and shorth methods ................................ 21

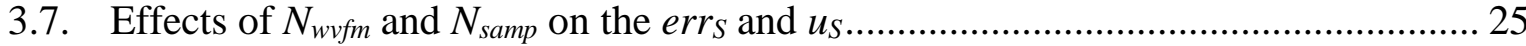

4. Conclusions.............................................................................................................................. 26

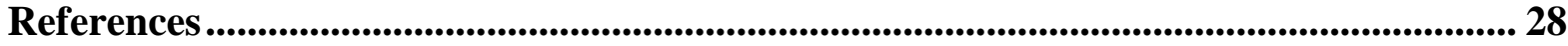

Annex A. Method for computing histogram mode values..................................................... 29

Annex B. Method for computing shorth values ........................................................................ 30

Annex C. Aberration generating functions ............................................................................... 31 


\section{List of Tables}

Table 1. Types of waveforms used in the simulations.

Table 2. Measurement uncertainty formulas used in the numerical simulation and comparison.

Table 3. Errors in the estimates of level(s) and their combined measurement uncertainties for the given estimators of level(s) for all of the different noise and aberration simulations performed. All values are given as percentage of level(s).

\section{List of Figures}

Fig. 1. Step-like waveform showing state levels, reference levels, reference level instants, waveform amplitude, and transition duration [from 1,2] .................................................. 1

Fig. 2. Plots of example waveforms. ......................................................................... 9

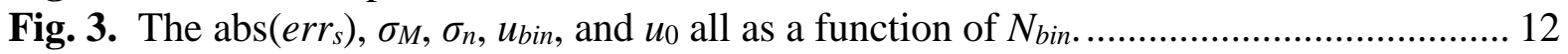

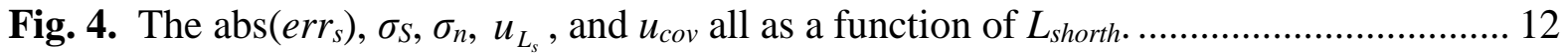

Fig. 5. Plot showing abs $\left\{e r r_{\mathrm{s}}\right\}$ for the different pairings of additive and multiplicated noise.

Fig. 6. Plot showing $u_{s}$ for different pairings of additive and multiplicated noise............... 14

Fig. 7. Plot of abs $\left\{\right.$ err $\left._{S}\right\}$ for the different sinusoidal aberration parameters and additive

noise levels, where err $\mathrm{s}$ is the error in level(s) for one of its four estimators (listed in the key).

Fig. 8. Plot of abs $\left\{e r r_{S}\right\}$ for the different sinusoidal aberration parameters and additive noise levels.

Fig. 9. The $u_{s}$ for the data shown in Fig. 7.

Fig. 10. The $u_{S}$ for the data shown in Fig. 8 .

Fig. 11. Plot of abs $\left\{e^{2} r_{S}\right\}$ for the different rectragular aberration parameters and additive noise levels, where err $r_{s}$ is the error in level(s) for one of its four estimators (listed in the key).

Fig. 12. The $u_{S}$ for the data shown in Fig. 11.................................................................. 20

Fig. 13. Plot of abs $\left\{\operatorname{err}_{S}\right\}$ for the different impulse-like aberration parameters and additive noise levels, where err is the error in level(s) for one of its four estimators (listed in the key)

Fig. 14. The $u_{s}$ for the data shown in Fig. 13. ............................................................... 22

Fig. 15. Distribution of errs for the different level estimators, where errs is given as a percentage of level( $\left(S_{r e f}\right)$.

Fig. 16. Distribution of $u_{s}$ for the different level estimators, where $u_{s}$ is given as a percentage of level(Sref).

Fig. 17. Plot of abs\{errs $\}$ for the different additive noise levels, different number of elements/samples in a waveform, and different number of waveforms averaged for the four

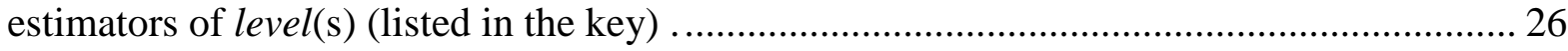

Fig. 18. The uncertainty in level(s) for the data shown in Fig. 17..................................... 27 


\section{Glossary}

The following terms and their definitions are taken from references [1] and [2].

level - "constant value having the same units as y,” $[1,2]$ where y refers to the signal being measured.

signal - "a physical phenomenon, one or more of whose characteristics may vary to represent information.” [1,2]

state - "particular level or, when applicable, particular level and upper and lower limits (the upper and lower state boundaries) that are referenced to or associated with that level" [1,2]

note 1: "multiple states are ordered from the most negative level to the most positive level, and the state levels are not allowed to overlap. The most negative state is called state 1 . The most positive state is called state $n$. The states are denoted by $s_{1}, s_{2}, \ldots$, $\mathrm{s}_{n}$; the state levels are denoted by level $\left(\mathrm{s}_{1}\right)$, level $\left(\mathrm{s}_{2}\right), \ldots$, level $\left(\mathrm{s}_{n}\right)$; the upper state boundaries are denoted by upper( $\left(\mathrm{s}_{1}\right)$, upper $\left(\mathrm{s}_{2}\right), \ldots$, upper $\left(\mathrm{s}_{n}\right)$; and the lower state boundaries are denoted by lower( $\left(\mathrm{s}_{1}\right), \operatorname{lower}\left(\mathrm{s}_{2}\right), \ldots$, lower $\left(\mathrm{s}_{n}\right) . ”[1,2]$

note 2: "States, levels, and state boundaries are defined to accommodate pulse metrology and digital applications. In pulse metrology, the levels of a waveform are measured and states (with or without associated state boundaries) are then associated with those levels. In digital applications, states are defined (with state boundaries) and the waveform values are determined to either lie within a state or not.” [1,2]

state occurrence - "contiguous region of a waveform that is bounded by the upper and lower state boundaries of a state, and whose duration equals or exceeds the specified minimum duration for state attainment. The state occurrence consists of the entire portion of the waveform that remains within the boundaries of that state" $[1,2]$

note 1: "State occurrences are numbered as ordered pairs $(s, n)$, where $s_{i}$ refers to the $i$ th state, and $n$ is the number of the occurrence of that particular state within the waveform epoch. In a given waveform epoch, when the waveform first enters a state $\mathrm{s}_{1}$, that state occurrence is $\left(\mathrm{s}_{1}, 1\right)$. If and when the waveform exits that state, that state occurrence is over. If and when the waveform next enters and remains in state $\mathrm{s}_{1}$, that state occurrence would be labeled $\left(\mathrm{s}_{1}, 2\right)$; and so on. Thus, the state occurrences for a single pulse are $\left(\mathrm{s}_{1}, 1\right),\left(\mathrm{s}_{2}, 1\right),\left(\mathrm{s}_{1}, 2\right)$. Note that a waveform can exit one state occurrence without (necessarily) immediately entering another state occurrence, that is, the waveform state between state occurrences can be undefined for some time interval, for example, during transitions and in the case of transients (such as, runt pulses).”[1,2]

waveform - "representation of a signal (for example, a graph, plot, oscilloscope presentation, discrete time series, equations, or table of values).” [1,2]

reference waveform - "waveform against which other waveforms are compared.” [1,2] 
waveform aberration - "algebraic difference in waveform values between all corresponding instants in time of a waveform and a reference waveform in a specified waveform epoch.” $[1,2]$

waveform epoch - "interval to which consideration of a waveform is restricted for a particular calculation, procedure, or discussion. Except when otherwise specified, the waveform epoch is assumed to be the span over which the waveform is measured or defined." [1,2] 


\section{Background}

State level is one of the most important waveform parameters in waveform metrology as it is common in the computation of almost all of the waveform parameters described in the International Electrotechnical Commission (IEC) 60469, "Transitions, pulses and related waveforms - Terms, definitions and algorithms" [1]; and the Institute of Electrical and Electronics Engineers (IEEE) Std-181, "IEEE Standard for Transitions, Pulses, and Related Waveforms" [2]. These standards list, define, and provide computational algorithms for a variety of parameters that are used to describe the characteristics of a waveform. The rationale for these waveform parameters standards is explained in the IEEE Std-181:

"..facilitates accurate and precise communication concerning parameters of transition, pulse, and related waveforms, and the techniques and procedures for measuring them. Because of the broad applicability of electrical pulse technology in the electronics industries, the development of unambiguous definitions for pulse terms, and the presentation of methods and/or algorithms for their calculation is important for communication between manufacturers and consumers within the electronics industry. The availability of standard terms, definitions, and methods for their computation helps improve the quality of products and helps the consumer better compare the performance of different products."

The documentary standard, IEC 62754, "Computation of Waveform Parameter Uncertainties," describes methods for the computation of waveform parameter measurement uncertainties [3]. The measurement uncertainty of state level affects the measurement uncertainty of all those state-level-dependent waveform parameters [4].

State level is the amplitude value associated with a given state, $\mathrm{s}_{i}$, where $i$ is a reference index. Therefore, $s_{i}$ refers to a constant-valued part of a waveform. However, for a region

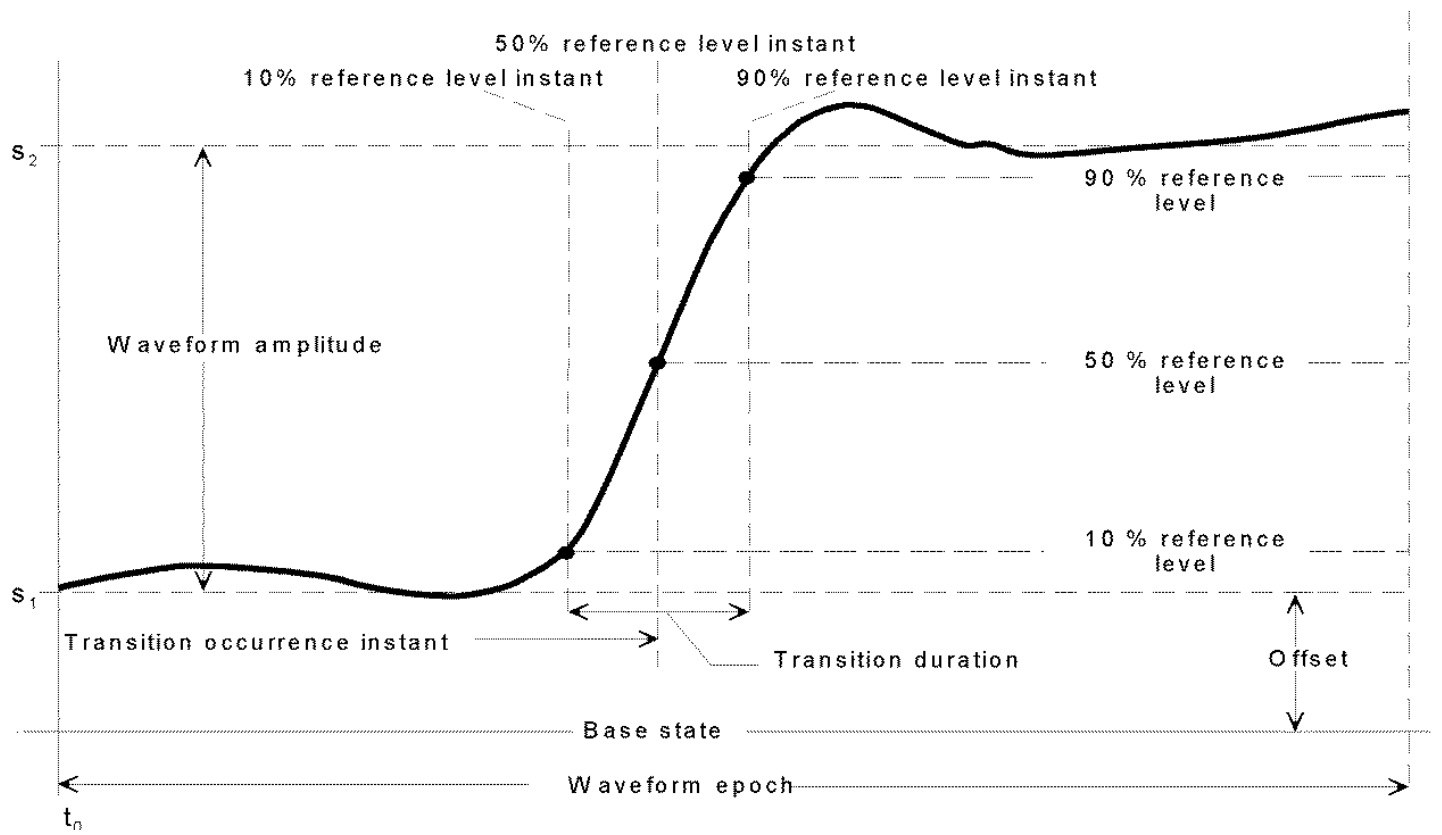

Fig. 1. Step-like waveform showing state levels, reference levels, reference level instants, waveform amplitude, and transition duration [from 1,2]. 
of a waveform to qualify as a state, it must meet the requirements for state occurrence [1,2]. The duration of $s_{i}$ is user-defined through the word "specified," and this influenced the type of waveforms used in this study (as will be described later in this section).

\subsection{Methods for determining level(s)}

The IEC 60469 and IEEE 181 describe several methods for obtaining level( $\left.\mathrm{s}_{i}\right)$, which are histogram, shorth, peak (maximum and minimum) magnitudes, initial and final states, other epochs, static levels, and user defined. Different entities participating in the development of these standard use the following methods but did not publish them, so technical references are not available for all. When these are not available, a brief description of an actual use case is given here. These methods may be used to determine level $\left(\mathrm{s}_{\mathrm{i}}\right)$ for the output of a pulse generator or of the impulse response or step response of a waveform recorder. A brief description of these methods is:

1) Histogram, mode - data-distribution based method. Application to pulse metrology can be found in [5].

2) Shorth [6] - data-distribution based method that was developed to approximate the histogram mode

3) Peak magnitudes: In this method, level( $\left.\mathrm{s}_{1}\right)$ is defined as the minimum value of the waveform and level $\left(\mathrm{s}_{2}\right)$ as the maximum value of the waveform. Assessment for pulse metrology can be found in [7].

4) Initial and final states: In this method, level( $\left.\mathrm{s}_{1}\right)$ is defined as the minimum of the initial (first) and final (last) waveform values and level( $\left.\mathrm{s}_{2}\right)$ as the maximum of the initial and final waveform values. Assessment for pulse metrology can be found in [7].

5) Other epochs: In this method, two or more waveform epochs are used, one epoch with a long duration to obtain level( $\left.\mathrm{s}_{1}\right)$ and level $\left(\mathrm{s}_{2}\right)$ of the waveform and the second epoch with a short duration to obtain the transition duration and other temporal parameters. The reason for the using the shorter-duration epoch is that the longer-duration epoch may not have the sampling rate to accurately compute the waveform parameters of transition duration, pulse duration, etc. NIST attempted such a process around 1998 to 2000 to improve the pulse measurement services it was providing. In this instance, a 70-GHz-analog-bandwidth, cryoelectronic, equivalent-time sampling oscilloscope provided the high-temporal resolution short-duration (less than 200 ps) epoch. This oscilloscope had an oscillatory low-frequency response and so it could not provide a reliable or accurate long-duration (to $10 \mathrm{~ns}$ ) epoch. A 20-GHz diode-based equivalent-time sampling oscilloscope provided the accurate long-duration epoch. A concern with this method is harmonization of the waveform's sampling rate and level $\left(\mathrm{s}_{1}\right)$ and level $\left(\mathrm{s}_{2}\right)$ alignments, both of which may impact the accuracy of the frequency spectrums obtained through conventional transforms.

6) Static levels: In this method, if the pulse generator can output static amplitude levels as well as a pulse, where these static levels are equal to level( $\left.\mathrm{s}_{1}\right)$ and level( $\left.\mathrm{s}_{2}\right)$ of its output pulse, then these static levels can be measured and used to estimate level( $\left.\mathrm{s}_{1}\right)$ and level( $\left.\mathrm{s}_{2}\right)$ of the pulse waveform. NIST used such a method until about 2003. The pulse generator used in this application had a toggle to switch between pulse output 
mode, static low to emulate level( $\left.\mathrm{s}_{1}\right)$, and static high to emulate level $\left(\mathrm{s}_{2}\right)$. A concern with this method is the accuracy of the static outputs to emulate level( $\left.\mathrm{s}_{i}\right)$.

7) User defined: In this method, the user specifies level( $\left.\mathrm{s}_{1}\right)$ and level( $\left.\mathrm{s}_{2}\right)$ based on previous experience and/or knowledge of the pulse generator. This has been used by seismic researchers who were members of the IEEE Subcommittee on Pulse techniques and who described measuring phenomenon occurring over very long (hours) epochs. In this case, previous knowledge and/or calibration data was used to specify level( $\left.\mathrm{s}_{i}\right)$.

Two of these methods, the histogram mode and the shorth, are based on the distribution of values contained in a given $s_{i}$. The histogram-mode method, when compared to the other statistical methods (central tendency measures), is the most commonly used method to compute level $\left(\mathrm{s}_{i}\right)$ because it a) approaches the expected value of level $\left(\mathrm{s}_{i}\right)$ for a large number of samples, b) is an intuitive method, c) is easy to compute, d) is not affected by a distribution that is skewed and asymmetric, whereas the median or mean are affected, and e) is not affected by values that deviate significantly from the expected value, whereas the mean is affected. The histogram-mode method is far more commonly used than others listed in IEC 60469 or IEEE 181 because a) of the greater reproducibility of the histogram-mode method than the methods 3) and 4), which are affected by noise and spurious signal content; no additional waveforms are required as in method 5), which can introduce errors in harmonizing waveform values if required; c) no special instrumentation is required as in method 6); and there is not subjective judgement as in method 7). However, there are instances in waveform metrology where the other methods listed in the IEC 60469 are applicable, such as unique single-occurrence events, long-duration events with short-duration transients, etc..

The shorth method was introduced in 2009 [8] for the computation of level $\left(\mathrm{s}_{i}\right)$ in pulse waveform metrology with the expectation that it would provide more accurate values of level $\left(\mathrm{s}_{i}\right)$ than the histogram-mode method and with smaller measurement uncertainties. This method has not gained significant popularity and is used primarily by a couple of national metrology institutes. This method was initially developed to approximate the histogram mode [6]. The shorth method is implemented by first reordering the values of the state into a nondecreasing sequence (see Annex B). This sequence is then reduced in length by removing values symmetrically from both ends of the sequence. For example, if the length, $L_{\text {shorth }}$, of the shorth interval is $0.6 N_{\text {samp }}$, where $N_{\text {samp }}$ is the number of values in the state, then $0.2 N_{\text {samp }}$ values at each end of the sequence are removed. This reduced-length reordered sequence is called the shorth. The average of the values of the shorth yields, in this case, level(s). In the extreme cases, when $L_{\text {shorth }}=N_{\text {samp }}$, the shorth equals the mean, and when $L_{\text {shorth }}$ is a minimum, the shorth equals the median.

\subsection{Overview}

In this paper, the estimation of level $\left(\mathrm{s}_{i}\right)$ and its measurement uncertainty, $u_{\mathrm{s}_{i}}$, as computed using the histogram-mode and shorth methods, are presented. This work continues with the initial examination [9] on the same subject in which the errors and uncertainties for all waveforms were aggregated. In this work, the errors and uncertainties are segregated to present the detailed effects of different aberrations and noise on the errors and measurement 
uncertainties. Comparisons of pulse parameter measurement methods, including level( $\left.\mathrm{s}_{i}\right)$, have been previously reported $[7,10]$. The effect of the histogram parameters on pulse parameters has also been examined [5].

In Section 2, the errors in the estimate of level( $\left.\mathrm{s}_{i}\right)$ and formulas for computing different measurement uncertainties, $u_{s}$, in level $\left(\mathrm{s}_{i}\right)$ for the shorth and histogram-mode methods are provided. The histogram-mode method is described in Sec. 2.1.1, followed by the shorth method in Sec. 2.1.2. Section 3 provides the detail of the comparison of the errors in the estimate of level $\left(\mathrm{s}_{i}\right)$ and the values of $u_{s}$ for these two methods for the different types of waveforms considered: effects of noise only are shown in Sec. 3.3, sinusoidal aberrations with varying level of noise are shown in Sec. 3.4, rectangular-pulse-train aberrations with varying level of noise are shown in Sec. 3.5, and impulse-like aberrations with varying level of noise are shown in Sec. 3.6. Section 3.7 provides a collective comparison for all noise and aberration for the two methods and Sec. 3.8 examines the effects of the number, $N_{\text {samp }}$, of samples in a waveform and the number, $N_{\text {wvfrm }}$, of waveforms used to create the waveform that was analyzed.

\section{Error and measurement uncertainty algorithms}

The reference waveform chosen for computing the errors and uncertainties in level( $\left.\mathrm{s}_{i}\right)$ is a constant-valued waveform. Noise and nominally realistic and representative waveform aberrations and noise are added to this reference waveforms. A constant-valued waveform was used for the reference waveform because:

1) The waveform values contained in transitions, pulses, etc., are not used in the computation of level( $\left.\mathrm{s}_{i}\right)$ and, therefore, serve no purpose for this study and, in some cases, may introduce errors.

2) Real (measured) waveforms exhibit asymptotic behavior at the starting and ending intervals of a waveform epoch. These intervals are used to compute level( $\left.\mathrm{s}_{i}\right)$ and, because of this asymptotic behavior, the true value of level $\left(\mathrm{s}_{i}\right)$ cannot be obtained. This asymptotic behavior is a result of the electronics of the pulse source and measuring instrument.

3) Simulated waveforms exhibit similar asymptotic behavior to b) but due to the mathematical models.

4) The state occurrence in real and simulated waveforms is user-specified, which may bias the values of level $\left(\mathrm{s}_{i}\right)$.

This section contains the algorithms used to compute errors and the measurement uncertainty, $u_{s}$, in level(s), where the subscript " $i$ " has been dropped for brevity. The formulas used to compute level(s) using the histogram-mode method are given in Annex A and for the shorth method in Annex B.

The error, err , in level(s) is computed using:

$$
\operatorname{err}_{\mathrm{s}}=\operatorname{level}\left(\mathrm{s}_{\mathrm{ref}}\right)-\operatorname{level}(\mathrm{s}) \text {, }
$$

where level( $\left(\mathrm{s}_{r e f}\right)$ is the value of the reference level. The reference waveform used had level $\left(\mathrm{s}_{\mathrm{ref}}\right)=1$. 
The level(s) in a typical waveform acquisition process may be estimated from a) an averaged waveform, that is, a waveform that is the result of the average of many independent and unique waveforms, or b) from the average of a set of level(s) values, where each value is taken from one of the independent and unique waveforms comprising the average waveform. These two options can yield different $u_{s}$ depending on whether the information from the individual waveforms comprising the average waveform is used. Consequently, there are four possible options for estimating level(s): two instantiations for the histogram-mode method and two for the shorth method. These four options can yield different results and so all will be considered in the analysis below. Furthermore, each of these options have two different methods for computing the uncertainty, $u_{s}$, in level(s), as will be described in this section.

\subsection{Mode method}

The mode method should be implemented using those methods described in [1 or 2] to ensure the appropriate number of histogram bins is selected. Two methods are given for computing level(s) using the mode values, which are given in 2.1.A and 2.1.B. For each of these two methods there are two possible ways to consider and subsequently compute measurement uncertainty.

\section{A. Average, $\bar{M}$, of modes}

The level(s) is obtained from the average level, $\overline{\text { level }}_{M}(\mathrm{~s})$, estimated from a set of mode values,

$$
\overline{\operatorname{level}}_{M}(\mathrm{~s})=\bar{M}=\frac{1}{N_{w v f m}} \sum_{i=1}^{N_{w f f m}} M\left\{W_{i}\right\}
$$

where $M\left\{W_{i}\right\}$ is the mode of the ith waveform, $W_{i}[t], N_{w v f m}$ is the number of waveforms used, and $[t]$ represents discrete time. Each $W_{i}[t]$ maybe the result of a single-shot (singlesweep) measurement or the average of many waveforms. Two different measurement uncertainty possibilities may be considered:

(1) the uncertainty, $\sigma_{M}$, in level(s) when knowledge of the $W_{i}[t]$, and its histograms, $H\left\{W_{i}\right\}$, is not known or used, is given by the standard deviation of the mode values.

(2) the uncertainty, $u_{M, W, 2}$, in level(s) when knowledge of the $W_{i}[t]$ and $H\left\{W_{i}\right\}$ is available and used, is given by:

$$
u_{M, W, 2}=\sqrt{\sigma_{M}^{2}+u_{H}^{2}},
$$

where $u_{H}$ represents the applicable histogram-based uncertainty contributions: 


$$
u_{H}=\frac{1}{N_{w v f m}} \sqrt{\sum_{i=1}^{N_{w v f m}} u_{H_{i}}^{2}}
$$

and

$$
u_{H_{i}}=\sqrt{u_{b i n, i}^{2}+u_{0, i}^{2}+\sigma_{n, i}^{2}} \text {. }
$$

The contributors to $u_{H_{i}}$ are now explained.

i. $u_{b i n}$

Each $W_{i}[t]$, because of noise, will have a different value for its extrema and, therefore, for the histogram bin width, $A_{b i n}$. These waveform-dependent bin widths, $A_{b i n, i}$, will affect $u_{b i n, i}$. The $y_{k}$, which is the $k$ th sample of the waveform, when assigned to a bin in the histogram, may have any value within the width of that bin. This means that the uncertainty associated with the value of that $y_{k}$ is uniformly distributed over the bin width. That uniform distribution can be approximated by a normal distribution, for the purpose of propagation of uncertainties, to give:

$$
u_{b i n, i}=\frac{A_{b i n, i}}{\sqrt{12}}
$$

ii. $u_{0}$

Each $W_{i}[t]$ will have a mode that is dependent on $N_{b i n}$, where $N_{b i n}$ is the number of bins for a given $H\left\{W_{i}\right\}$. For each $W_{i}[t]$, there is an uncertainty, $u_{0, i}$, associated with the selection of $N_{b i n}$. The $u_{0, i}$ is given by:

$$
u_{0, i}=\sqrt{\frac{1}{\left(N_{b i n, \text { range }}-1\right)} \sum_{j=N_{\text {bin }, l o}}^{N_{\text {bin }, h i}}\left(M_{r e f}-M\left\{W_{j}\right\}\right)^{2}},
$$

and

$$
N_{\text {bin, range }}=N_{b i n, h i}-N_{b i n, l o},
$$

where $M_{\text {ref }}$ is the mode for the default number, $N_{b i n, r e f}$, of bins which was set to $10, j$ is the counter for $N_{b i n}$, and $N_{b i n, h i}$ and $N_{b i n, l o}$ are user-defined upper and lower limits to the number of bins. The $N_{b i n, h i}$ and $N_{\text {bin,lo }}$ should be selected so that $N_{\text {bin,range }}$ demonstrates a change in level(s) with a change in $N_{b i n}[5]$ and as demonstrated in Fig. 4.

iii. $\sigma_{n}$ Each $W_{i}[t]$ will have a unique noise distribution represented by $\sigma_{n, i}$.

B. Mode of average waveform, $\bar{W}[t]$ 
The level(s) is obtained from the estimated level, level $_{\bar{M}}(\mathrm{~s})$, of the mode of the averaged waveform, $\bar{W}[t]$,

$$
\operatorname{level}_{\bar{M}}(\mathrm{~s})=M\{\bar{W}[t]\}
$$

Two different measurement uncertainty possibilities may be considered:

(1) the uncertainty, $u_{M, \bar{W}, 1}$, in level(s) when knowledge of the $W_{i}[t]$ and $H\left\{W_{i}\right\}$ is not available or used but knowledge of $\bar{W}[t]$ and $H\{\bar{W}\}$ is used, is given by:

$$
u_{M, \bar{W}, 1}=\sqrt{u_{b i n}^{2}+u_{0}^{2}+\sigma_{n}^{2}}
$$

where $u_{b i n}$, described by (6), $u_{0}$, described by (7), and $\sigma_{n}$ are used as applied to $\bar{W}[t]$ (that is, the waveform subscript $i$ can be ignored).

(2) the uncertainty, $u_{M, \bar{W}, 2}$, in level(s) when knowledge of the $W_{i}[t]$ and $H\left\{W_{i}\right\}$ is available and used is given by the following estimate:

$$
u_{M, \bar{W}, 2}=\sqrt{u_{M, \bar{W}, 1}^{2}+u_{H}^{2}},
$$

where $u_{M, \bar{W}, 1}$ is described by (10) and $u_{H}$ by (4). There is no obvious functional relationship between the histogram parameters of $\bar{W}[t]$ and those of $W_{i}[t]$.

\subsection{Shorth method}

The shorth method should be implemented using those methods described in [1 or 2]. Two methods are given for computing level(s) using the shorth values, which are given in 2.2.A and 2.2.B. For each of these two methods there are two possible ways to consider and subsequently compute measurement uncertainty.

A. Average, $\bar{S}$, of the shorths

The level(s) is obtained from the average level, $\overline{\text { level }}_{s}(\mathrm{~s})$, estimated from a set of shorth values,

$$
\overline{\operatorname{level}}_{S}(\mathrm{~s})=\bar{S}=\frac{1}{N_{w v f m}} \sum_{i=1}^{N_{w v f m}} S\left\{W_{i}\right\}
$$

where $S\left\{W_{i}\right\}$ is the shorth of the ith waveform, $W_{i}[t]$. Each $S\left\{W_{i}\right\}$ maybe the result of a single-shot (single-sweep) measurement or the average of many waveforms. Two different measurement uncertainty possibilities may be considered:

(1) the uncertainty, $\sigma_{S}$, in level(s) when knowledge of the $W_{i}[t]$ is not available or used is given by the standard deviation of the shorth values. 
(2) the uncertainty, $u_{S, W, 2}$, in level(s) when knowledge of the $W_{i}[t]$ and $S\left\{W_{i}\right\}$ is available and is given by:

$$
u_{S, W, 2}=\sqrt{\sigma_{S}^{2}+u_{\mathrm{cov}}^{2}+u_{L_{S}}^{2}}
$$

where $u_{L_{S}}$ is the uncertainty in the length, $L_{\text {shorth }}$, of the shorth interval and $u_{c o v}$ is computed from the covariance matrix (see (16)). The $u_{c o v}$ is not a necessity for computing $u_{s}$ when using the shorth method, however, $u_{\text {cov }}$ should be included for a complete and rigorous computation of $u_{s}$. Each waveform will have different values that will influence the shorth because of the waveform-independent noise. These waveforms may each have a unique shorth-length uncertainty, $u_{L_{S}, i}$, due to the selection of the shorth interval. The $u_{L_{S}}$ is given by

$$
u_{L_{S}}=\frac{1}{N_{w v f m}} \sqrt{\sum_{i=1}^{N_{w v f m}} u_{L_{S}, i}^{2}}
$$

where

$$
u_{L_{S, i}}=\sqrt{\frac{1}{\left(L_{\text {shorth }, h i}-L_{\text {shorth }, o}\right)} \sum_{j=L_{\text {shorth }, o}}^{L_{\text {shorth hi }}}\left(S_{r e f}-S_{i, j}\right)^{2}} .
$$

and $L_{\text {shorth,hi }}$ is the longest length of the shorth interval used to compute $u_{L_{S}}, L_{s h o r t h, l o}$ is the shortest length of the shorth interval used to compute $u_{L_{S}}, S_{r e f}$ is level(s) for the default length of the shorth interval, and $S_{j}$ is level(s) for the jth length of the shorth interval. The $L_{s h o r t h, h i}$

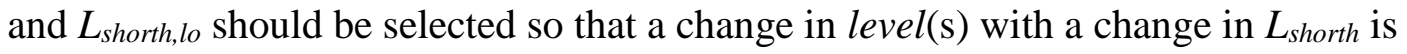
demonstrated, as shown in Fig. 3.

The $u_{\text {cov }}$ is given by

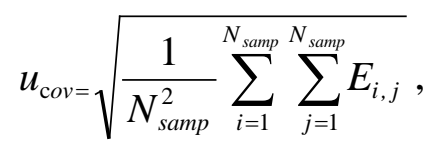

and $N_{\text {samp }}$ is the number of samples in a waveform, which is the same for each ith waveform,

$$
E_{i, j}=\frac{1}{N_{w v f m}-1} \sum_{k=1}^{N_{w v f m}}\left(y_{k, i}-\mu_{y_{i}}\right)\left(y_{k, j}-\mu_{y_{j}}\right)
$$

$y_{i, j}$ is the $i^{\text {th }}$ sample of the $j^{\text {th }}$ waveform, and 


$$
\mu_{y_{i}}=\frac{1}{N_{w v f m}} \sum_{j=1}^{N_{w f m}} y_{i, j}
$$

B. Shorth of average waveform, $\bar{W}[t]$

The level(s) is obtained from the estimated level, $\operatorname{level}_{\bar{S}}(\mathrm{~s})$ of the shorth of $\bar{W}[t]$,

$$
\operatorname{level}_{\bar{S}}(\mathrm{~s})=S\{\bar{W}\}
$$

Two different measurement uncertainty possibilities may be considered:

(1) the uncertainty, $u_{s, \bar{W}, 1}$, in level(s) when knowledge of the $W_{i}[t]$ and $S\left\{W_{i}\right\}$ is not available or used is given by:

$$
u_{S, \bar{W}, 1}=\sqrt{u_{L_{S}}^{2}+\sigma_{n}^{2}},
$$

where $u_{L_{s}}$, described by (14), and $\sigma_{n}$ are used as applied to $\bar{W}[t]$, (that is, the waveform subscript $i$ can be ignored).

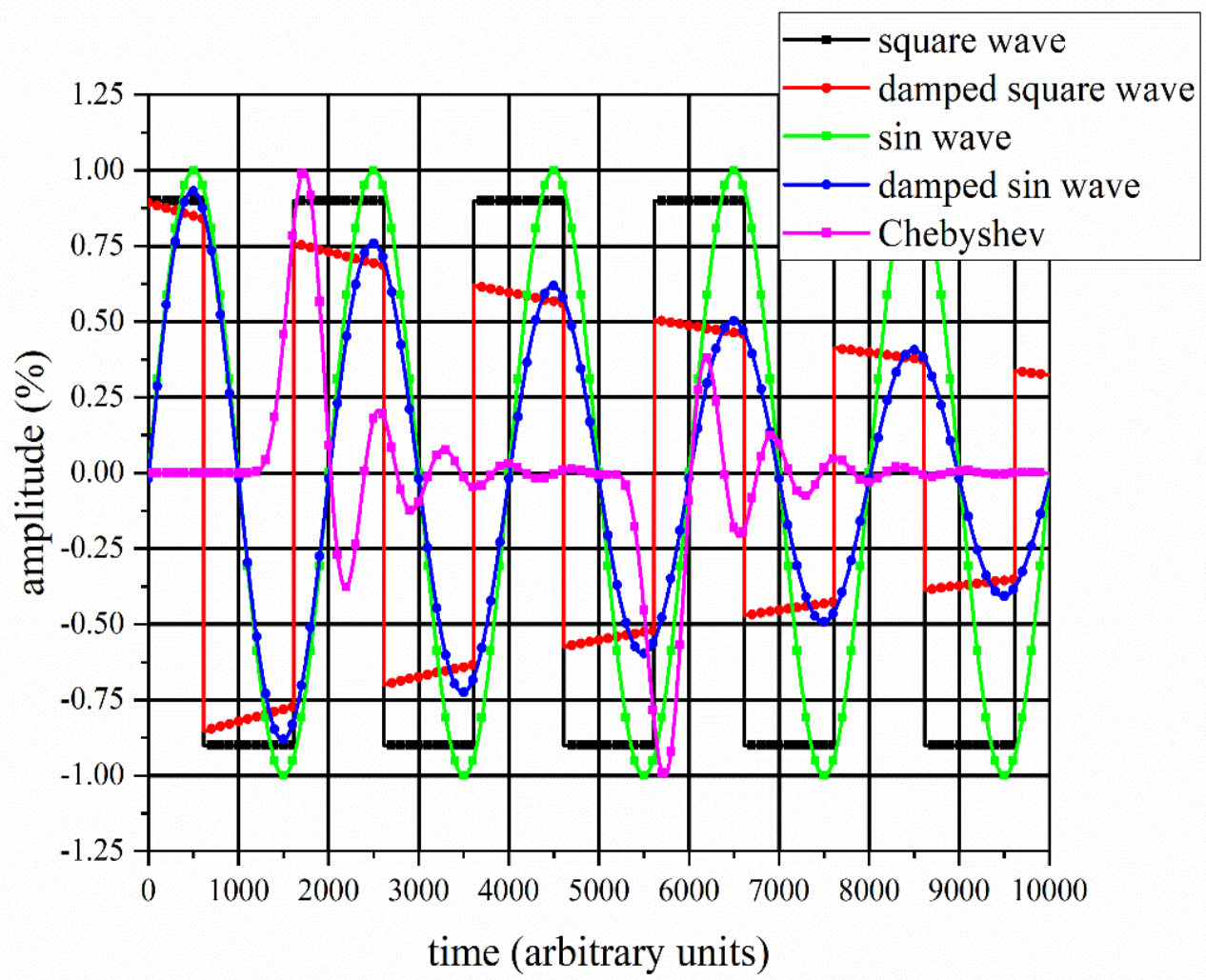

Fig. 2. Plots of example waveforms.

Vertical and horizontal scaling has been adjusted to facilitate viewing. 
(2) $u_{S, \bar{W}, 2}$ : the uncertainty in level(s) when knowledge of the $W_{i}[t]$ and $S\left\{W_{i}\right\}$ is available and used is given by:

$$
u_{S, \bar{W}, 2}=\sqrt{u_{S, \bar{W}, 1}^{2}+u_{c o v}^{2}},
$$

where $u_{s, \bar{w}, 1}$ is described by (20) and $u_{c o v}$ by (16).

\section{Computation and comparison of level(s) and $u_{s}$}

Numerical experiments were performed by adding noise (additive, multiplicative, and both) and various periodic and aperiodic aberrations to level(s $\left.\mathrm{s}_{\mathrm{r} f}\right)$. All noise and aberrations are zero mean. Table 1 lists the types of noise and aberrations that were used in this work. The types of aberrations were selected to represent the range and type of aberrations observed in waveforms and that could be easily simulated and reproduced. Normally-distributed white noise was used because that is the typical and largest noise component observed in

Table 1. Types of waveforms used in the simulations.

\begin{tabular}{|c|c|c|c|c|}
\hline Waveforms & Parameters & \multicolumn{3}{|c|}{ Value } \\
\hline \multicolumn{5}{|l|}{ Reference } \\
\hline & constant value & \multicolumn{3}{|c|}{1} \\
\hline & 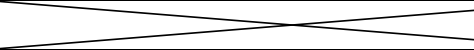 & \multicolumn{3}{|c|}{$\mathrm{N}_{2}$} \\
\hline \multicolumn{5}{|l|}{ Aberrations (zero mean) } \\
\hline \multirow[t]{4}{*}{$\begin{array}{c}\text { impulsive (Chebyshev } \\
\text { polynomial) }\end{array}$} & ripple & \multicolumn{3}{|c|}{$0.001 \mathrm{~dB}$} \\
\hline & cutoff frequency & 0.1 & 0.01 & 0.001 \\
\hline & polynomial order & 2 & 4 & 8 \\
\hline & Noise*, rms amplitude (\%) & 0 & 1 & 10 \\
\hline \multicolumn{5}{|l|}{ sinusoidal } \\
\hline & magnitude (\%) & 10 & 100 & \\
\hline & number of cycles & $N / 2000$ & $N / 100$ & \\
\hline & exponential decay , e(-x) & 0 & $5 / N$ & $20 / N$ \\
\hline & Noise*, rms amplitude (\%) & 0 & 1 & 10 \\
\hline \multicolumn{5}{|l|}{ rectangular } \\
\hline & amplitude (\%) & 1 & 10 & \\
\hline & number of cycles & $N / 2000$ & & \\
\hline & duty factor & 0.5 & 0.80 & \\
\hline & exponential decay , e(-x) & $5 / N$ & & \\
\hline & Noise*, rms amplitude (\%) & 0 & 1 & 10 \\
\hline & & $\sum<$ & $\geq<$ & $2<$ \\
\hline \multicolumn{5}{|l|}{ Noise (zero mean) } \\
\hline additive, normal, white & rms amplitude (\%) & 0 & 1 & 10 \\
\hline multiplicative, normal, white & rms amplitude (\%) & 0 & 1 & 10 \\
\hline
\end{tabular}

*Noise used was normally-distributed additive white noise. 
waveforms. The low levels of noise and aberration amplitudes $\left(\leq 0.02\right.$ level $\left.\left(\mathrm{s}_{\text {ref }}\right)\right)$ used here are typically observed in the states of step-like and impulse-like waveforms where waveform averaging is used in the waveform acquisition process. The high levels of noise and aberration amplitudes $\left(0.1\right.$ level $\left.\left(\mathrm{s}_{\mathrm{r} e f}\right)\right)$ were used to assess the robustness of the different methods to this level of noise and aberration. These aberrations on a signal may arise from coupling between the signal line (of interest) and adjacent lines (carrying other signals but not the one being measured), internal coupling of clock or strobe signals onto the signal line of interest, electromagnetic interference, etc. Figure 2 shows the types of waveforms used, where the scales have been changed to facilitate viewing. The formulas for the aberrations generated are given in Annex C.

The data presented here show the estimates of $u_{s}$ and $e r r_{s}$ for the histogram-mode and shorth methods and segregate the results of the different noise types and amplitudes and different aberrations with and without noise. This is different from the study described in [9] where the results of all the different types of waveforms aberrations and levels of noise were aggregated to show the common distributions of $e r r_{s}$ and $u_{s}$ for all cases. Although four possible methods were considered in Sec. 2.1 for estimating level(s), and for each of these methods two different methods for computing $u_{S}$ were given, only the most metrologically conservative methods are considered further. These are the four $u_{s}$ computational methods that consider all possible uncertainty contributors, including those from $W_{i}[t]$. These four methods are shown in Table 2.

Each waveform used in this analysis contained a number, $N_{\text {samp }}=10000$, of samples (elements), and each average waveform comprised a number, $N_{w v f m}=1000$, of independent noisy waveforms. The seed for the random number generator that was used to create each noisy waveform was unique. Each simulation run used 1000 averaged waveforms, effectively emulating 1000 unique measurements. The distributions for $e r r_{s}$ and $u_{s}$ for each simulation using the same waveform-aberration parameter settings but with different noise was observed to be nominally the same for each simulation (data not shown).

Table 2. Measurement uncertainty formulas used in the numerical simulation and comparison.

\begin{tabular}{|l|c|c|c|}
\hline \multicolumn{1}{|c|}{ Method } & Formula for level(s) & $\begin{array}{c}\text { Measurement } \\
\text { Uncertainty Formula }\end{array}$ & $\begin{array}{c}\text { Uncertainty } \\
\text { Equation }\end{array}$ \\
\hline $\begin{array}{l}\text { Histogram } \\
\text { mode }\end{array}$ & $\overline{\operatorname{level}}_{M}(\mathrm{~s})=\bar{M}=\frac{1}{N_{w v f m}} \sum_{i=1}^{N_{\mathrm{wvfm}}} M\left\{W_{i}\right\}$ & $u_{M, W, 2}=\sqrt{\sigma_{M}^{2}+u_{H}^{2}}$ & 3 \\
\hline $\begin{array}{l}\text { Histogram } \\
\text { mode }\end{array}$ & level $_{\bar{M}}(\mathrm{~s})=M\{\bar{W}\}$ & $u_{M, \bar{W}, 2}=\sqrt{u_{M, \bar{W}, 1}^{2}+u_{H}^{2}}$ & 11 \\
\hline Shorth & $\overline{\operatorname{level}}_{S}(\mathrm{~s})=\bar{S}=\frac{1}{N_{w v f m}} \sum_{i=1}^{N_{w v f m}} S\left\{W_{i}\right\}$ & $u_{S, W, 2}=\sqrt{\sigma_{S}^{2}+u_{\mathrm{cov}}^{2}+u_{L_{S}}^{2}}$ & 13 \\
\hline Shorth & $\operatorname{level}_{\bar{S}}(\mathrm{~s})=S\{\bar{W}\}$ & $u_{S, \bar{W}, 2}=\sqrt{u_{S, \bar{W}, 1}^{2}+u_{c o v}^{2}}$ & 21 \\
\hline
\end{tabular}



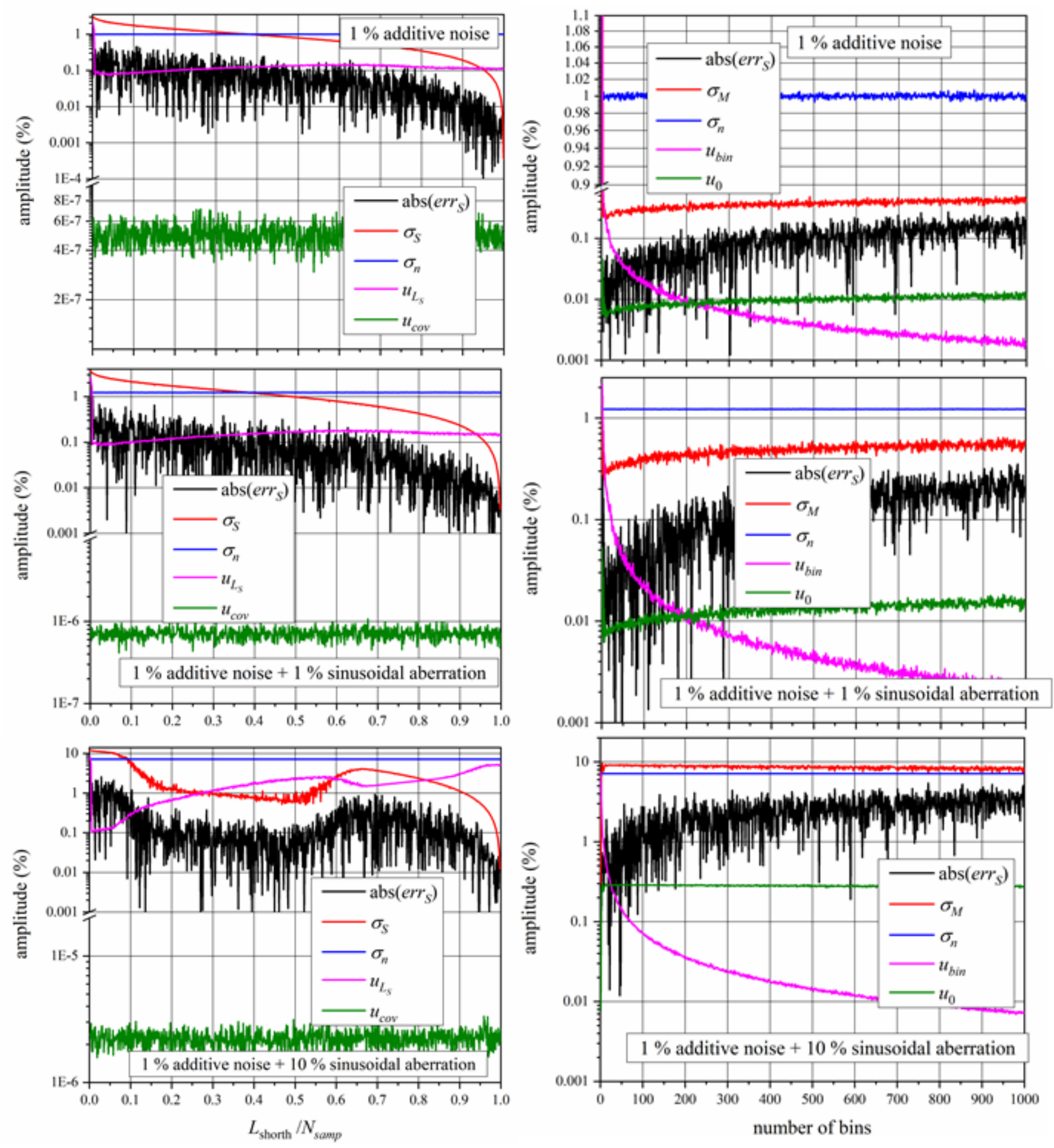

Fig. 4. The abs(errs $), \sigma_{S}, \sigma_{n}, u_{L_{s}}$, and $u_{c o v}$ all as a function of $L_{\text {shorth }}$.

Fig. 3. The abs $\left(e r r_{s}\right), \sigma_{M}, \sigma_{n}, u_{b i n}$, and $u_{0}$ all as a function of $N_{b i n}$. 


\section{1. $\quad$ Selection of $\boldsymbol{L}_{\text {shorth,ref }}$ and $N_{\text {bin,ref }}$}

The selection of $L_{\text {shorth, ref }}$ and $N_{\text {bin,ref }}$ affects $u_{s}$. The waveform used to examine the selection of $L_{\text {shorth,ref }}$ and $N_{\text {bin,ref }}$ had normally-distributed white noise with $\sigma_{n}=0.01$ level(sref). In Figure 3, abs(errs) for the shorth method is computed using:

$$
\operatorname{abs}\left(\operatorname{err}_{\mathrm{S}}\right)=\left|\operatorname{level}\left(\mathrm{s}_{\text {ref }}\right)-\overline{\operatorname{level}}_{S}(\mathrm{~s})\right| .
$$

For this waveform, the largest contributors to $u_{S}$ for smallamplitude aberrations is $\sigma_{n}$ and $\sigma_{s}$ (as defined in (16)). As the amplitude of the aberration increases, $u_{L_{S}}$ also becomes a significant contributor. Typically, waveform parameter uncertainties are computed in applications where aberration amplitudes are small and, in these cases, $u_{S}$ will be dominated by $\sigma_{n}$ and $\sigma_{S}$. As can be seen in Fig. 3 , abs $\left(e r r_{s}\right)$ and $\sigma_{S}$ nominally decrease with increasing $L_{\text {shorth }}$ whereas $u_{L_{\varsigma}}$ nominally increases with increasing $L_{\text {shorth. }}$ However, all three of these curves have a nominally small slope over the $L_{\text {shorth }}$ range of about $0.3 N_{\text {samp }}$ to 0.7 $N_{\text {samp}}$, except for the extreme case of $10 \%$ sinusoidal aberration. Based on these results, the selection of $L_{\text {shorthref }}=0.5 N_{\text {samp }}$ is not contraindicated but may not be optimal. Since the only researchers actively using the shorth method in pulse waveform metrology [6] set $L_{\text {shorth, } r e f}=0.5 N_{\text {samp }}$, the use of

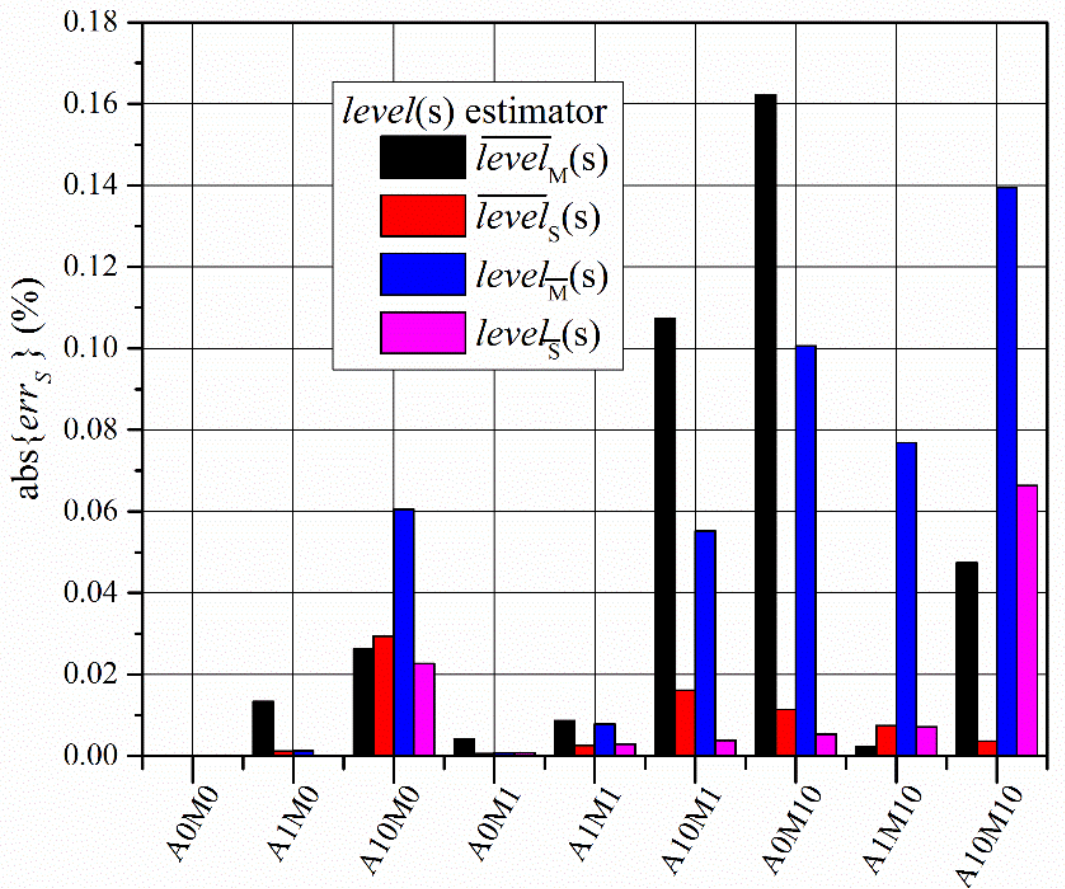

Fig. 5. Plot showing abs $\left\{e r r_{\mathrm{s}}\right\}$ for the different pairings of additive and multiplicated noise.

The err $r_{s}$ is the error in level(s) for one of its four estimators (listed in the key). Horizontal annotation:

- A0, A1, A10 - additive rms noise amplitude was set to $0 \%, 1 \%$, or $10 \%$ of level( Sref $)$.

- M0, M1, M10 - multiplicative rms noise amplitude was set to $0 \%, 1 \%$, or $10 \%$ of level(Sref).

$L_{\text {shorth,ref }}=0.5 N_{\text {samp }}$ as the reference was adopted here. For the simulations, the lower limit, $L_{\text {shorth,lo, }}$, to the shorth length was set to $0.01 N_{\text {samp }}$ and the upper limit, $L_{\text {shorth,hi, was set to } 1.0}$ $N_{\text {samp }}$

The abs(errs) for the histogram-mode method shown in Fig. 4 is compute using

$$
\operatorname{abs}\left(\operatorname{err}_{S}\right)=\left|\operatorname{level}\left(\mathrm{s}_{\text {ref }}\right)-\overline{\operatorname{level}}_{M}(\mathrm{~s})\right| .
$$


The dominant uncertainty contributors to $u_{S}$ (see Fig. 4) for the histogram-mode method are $\sigma_{n}$ and $\sigma_{M}$ for $N_{b i n} \geq 10$.

Consequently, there is no advantage for increasing $N_{b i n}$ beyond 10 in this study where the waveform is defined as a constant level, level(sref). For non-constantvalued waveforms (which are realistically encountered), $N_{b i n}$ should be adjusted to appropriately meet the measurement uncertainty requirements. For the simulations performed here, the lower limit, $N_{b i n, l o}$, for the number of bins was set to 10 and the upper limit, $N_{b i n, h i}$, was set to 1000 .

When these simulations are repeated, the relationship between the different uncertainty contributions for the histogram-mode and shorth methods do not change

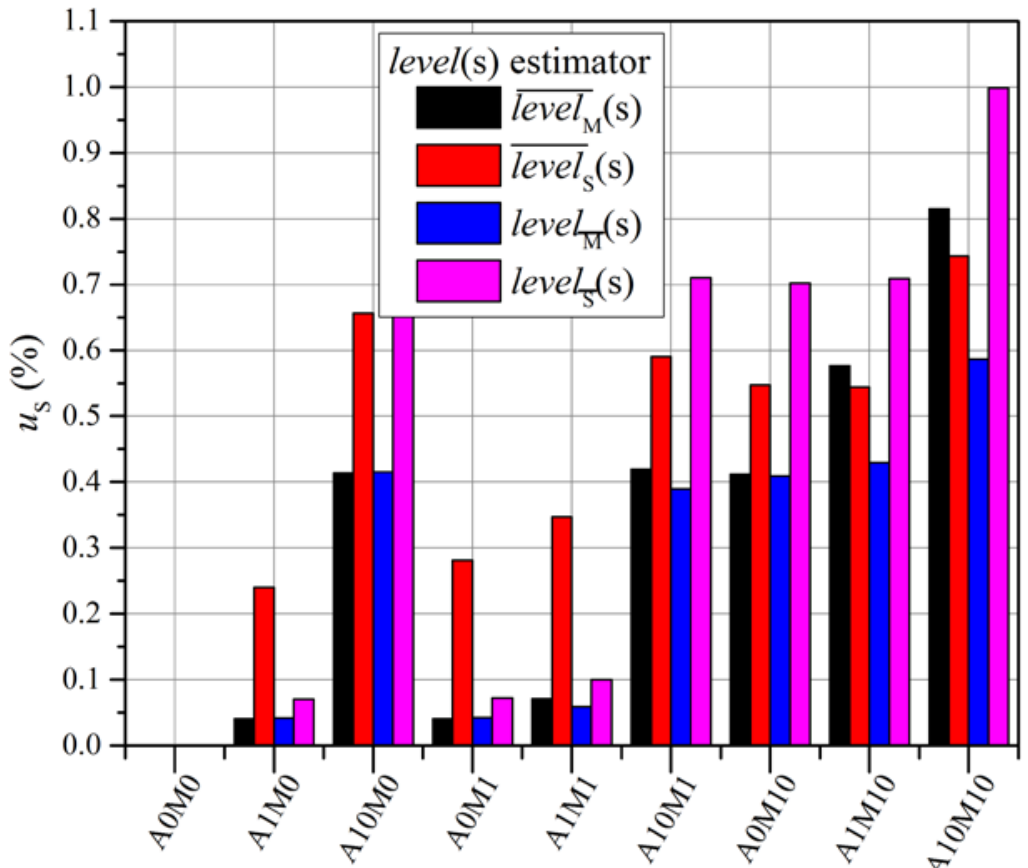

Fig. 6. Plot showing $u_{s}$ for different pairings of additive and multiplicated noise.

(See caption in Fig. 5 for an explanation of horizontal axis annotation.) (data not shown), thus indicating that the number of elements, waveforms, and simulation runs are sufficient for this study.

\subsection{Comparison of histogram-mode and shorth methods for noisy waveforms with no aberrations}

The graphs in Figs. 5 and 6 show errs and $u_{s}$. The shorth method typically provides estimates of level(s) that are more accurate than those provided by the histogram-mode method (see Fig. 5) for additive and multiplicative amplitude noise. The combined uncertainties (see Fig. 6), however, are typically greater for the shorth method. For additive and multiplicative noise, there is no obvious advantage in $u_{s}$ between the average of the modes (or shorths) over the mode (or shorth) of the average. More importantly, for either method, the relative magnitude of $u_{s}$ observed here ( $<1 \%$ of waveform amplitude) is typically less than the amplitude noise observed in waveforms acquired from real signals [10].

\subsection{Comparison of histogram-mode and shorth methods for noisy waveforms with sinusoidal aberrations}

Figures 7 through 10 compare $u_{s}$ and the abs $\left(e r r_{s}\right)$ for sinusoidal aberrations plus additive noise. In this case, the shorth method generally provides more accurate estimates for level(s) 
than did the histogram-mode method, as shown by Figs. 7 and 8. Similarly, the shorth method provides smaller $u_{S}$ than does the histogram-mode method (see Figs. 9 and 10).

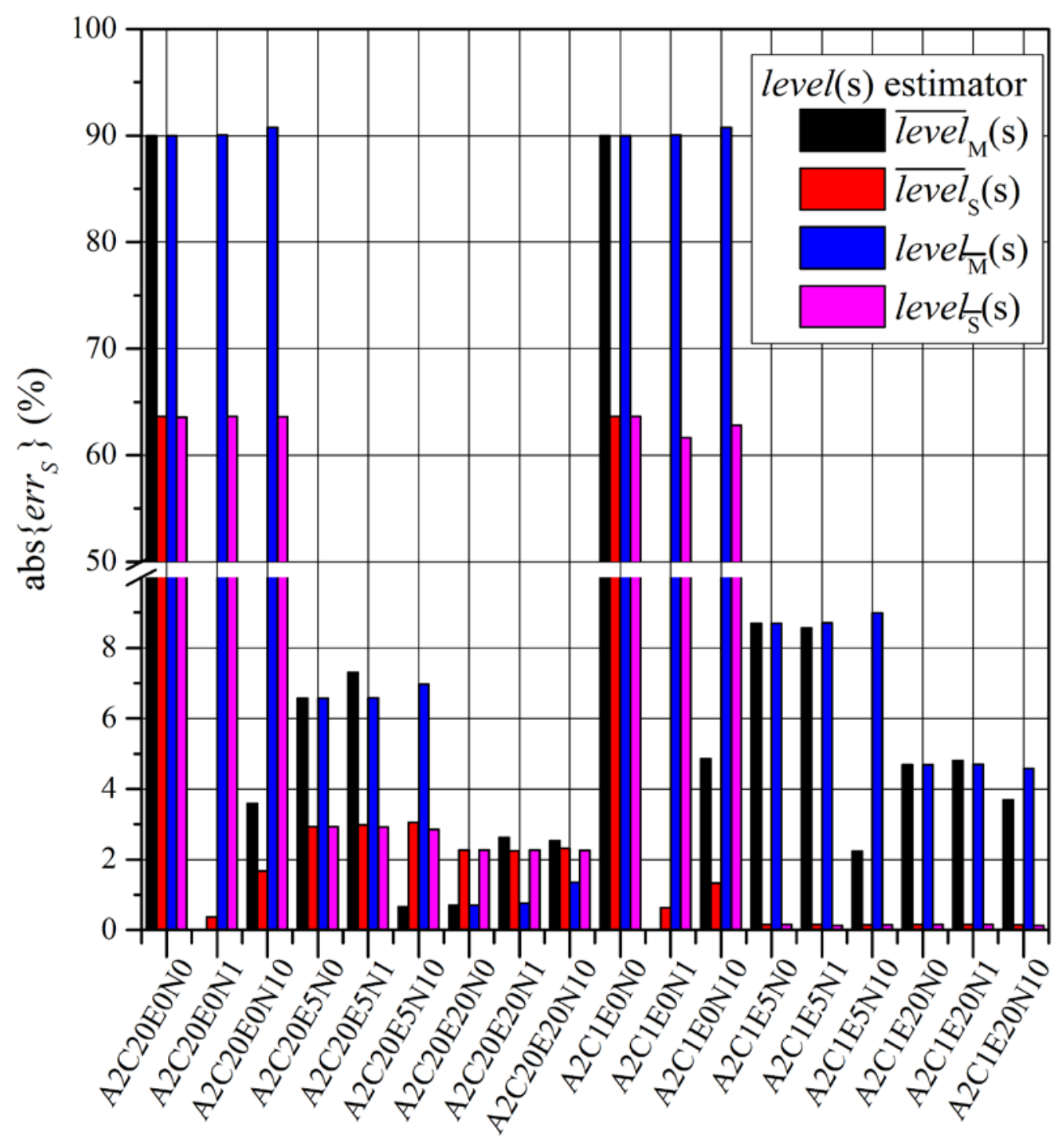

Fig. 7. Plot of abs $\{$ errs $\}$ for the different sinusoidal aberration parameters and additive noise levels, where err $r_{s}$ is the error in level(s) for one of its four estimators (listed in the key).

Horizontal annotation:

- A1, A2 - sinewave magnitude was set to $10 \%$ or $100 \%$ of level(sref)

- C1, C2 - number of sinewave periods was set to 5 or 100

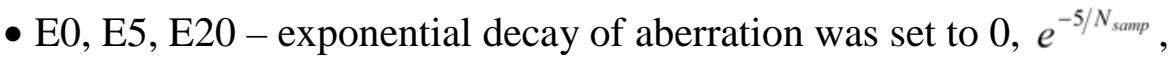
or $e^{-20 / N_{\text {samp }}}$

- N0, N1, N10 - rms noise amplitude was set to $0 \%, 1 \%$, or $10 \%$ of level(sref). 
Although a waveform aberration magnitude of $100 \%$ of level(s) is not likely in most measurement applications, these simulations show the inability of either method to reliability estimate level(s) with extreme waveform aberrations. The results also indicate that estimates of level(s) with sinewave aberrations at the higher frequency is more accurate than that at the lower frequency, but that $u_{s}$ is nominally the same for both frequencies. The effect of the exponential decay shows a decrease in abs $\left\{e r r_{s}\right\}$ and $u_{S}$ with increasing decays for both methods. This is expected based on the reduction of the influence of the waveform aberration on the waveform.

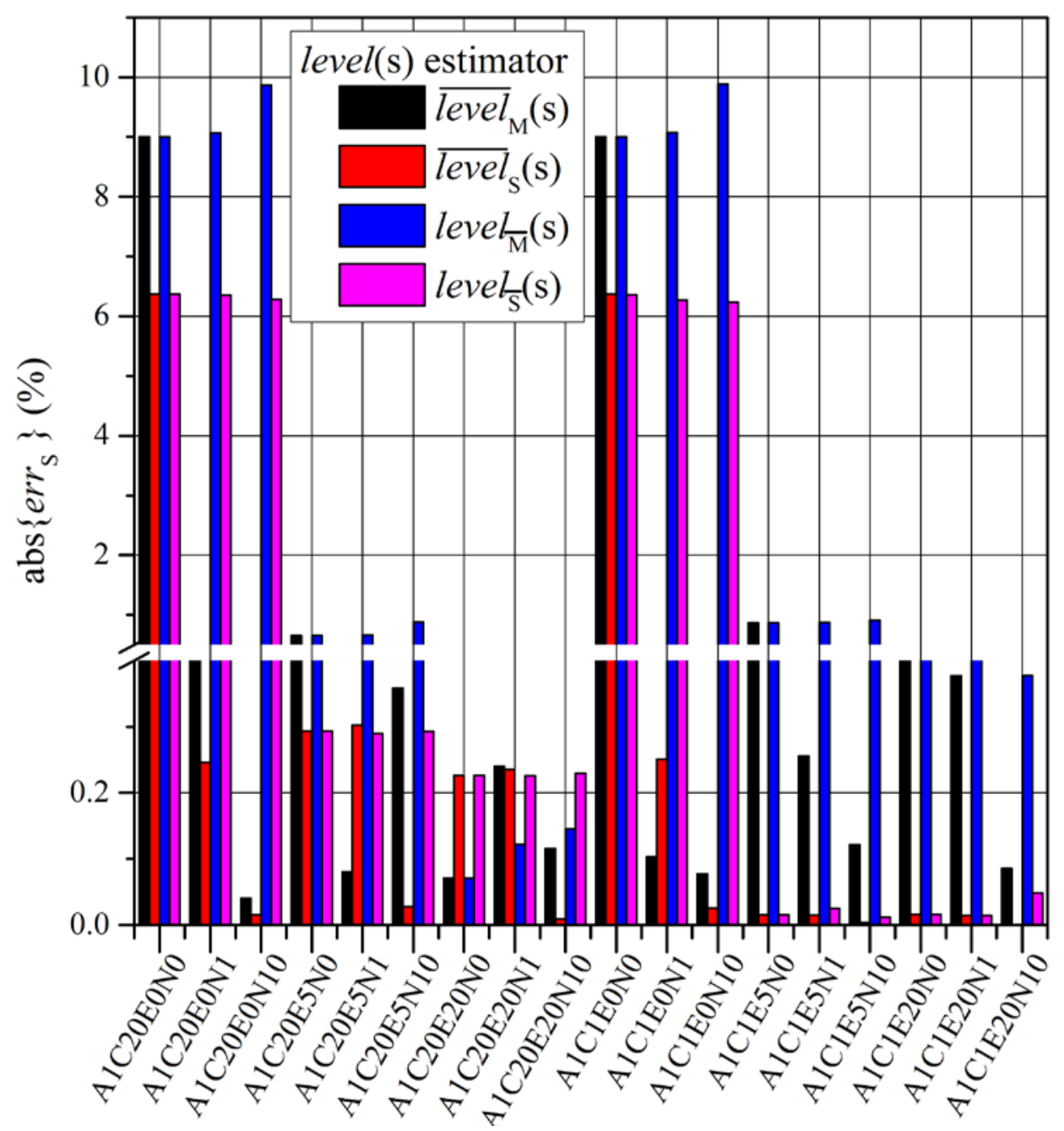

Fig. 8. Plot of abs\{errs $\}$ for the different sinusoidal aberration parameters and additive noise levels.

This is similar to the data presented in Fig. 7 but with different values of sinusoidal aberration parameters the key and horizontal axis annotation is the same as that in Fig. 7. 


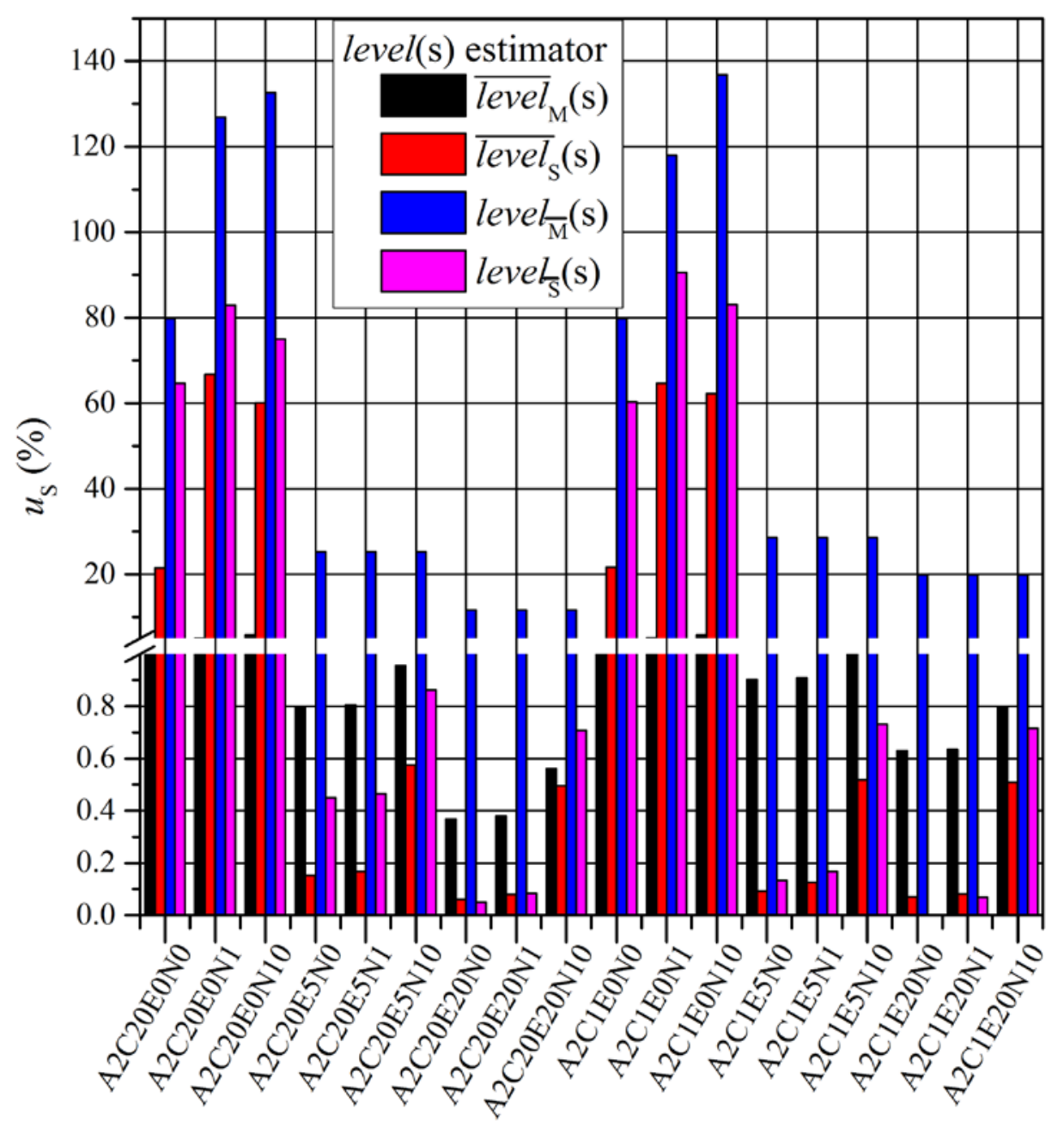

Fig. 9. The $u_{s}$ for the data shown in Fig. 7. 


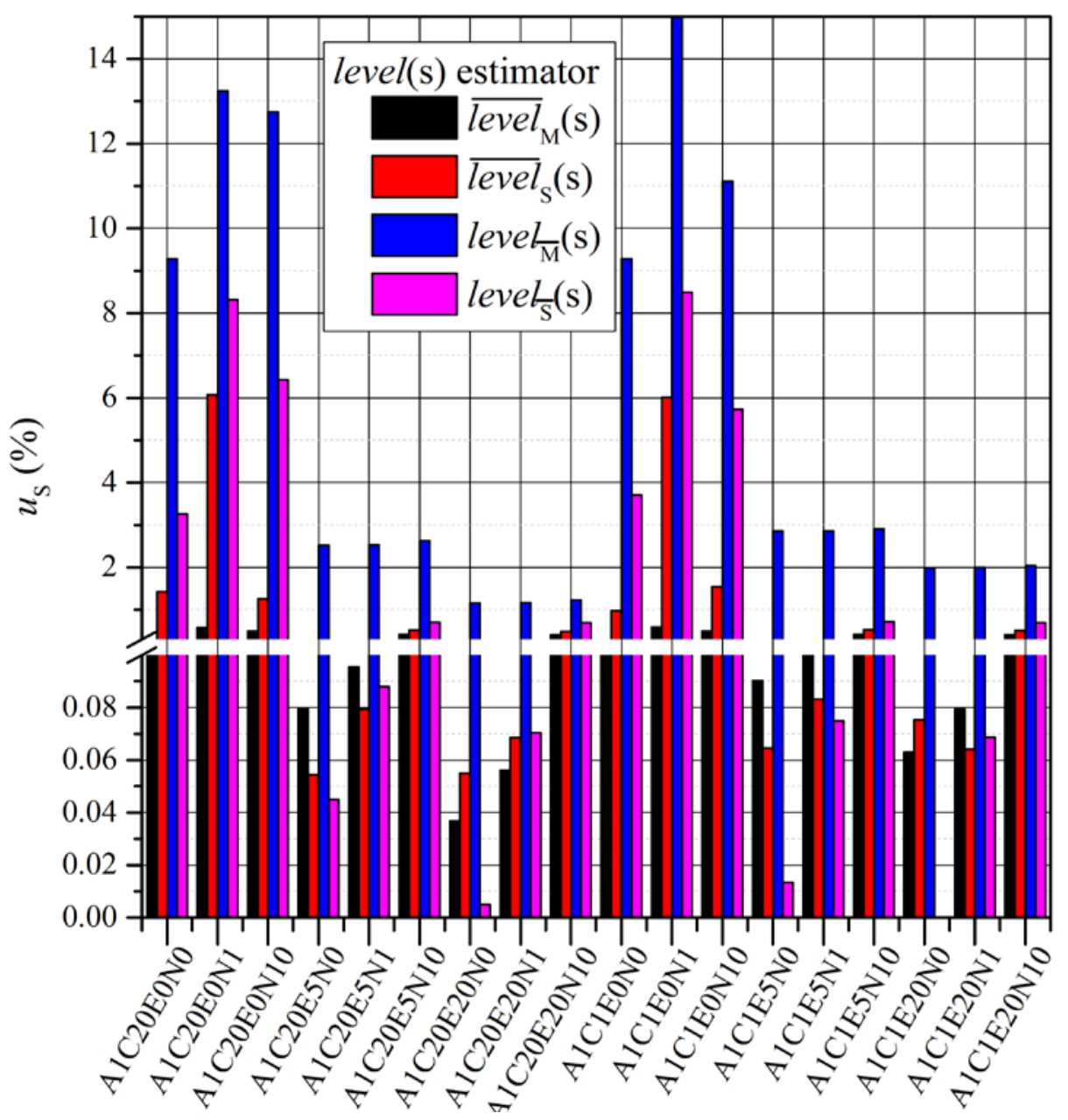

Fig. 10. The $u_{s}$ for the data shown in Fig. 8. 


\subsection{Comparison of histogram-mode and shorth methods for noisy waveforms with rectangular-pulse-train aberrations}

The effects of rectangular-pulse-train aberration with and without noise on errs and $u_{s}$ are examined here (see Figs. 11 and 12). Based on the results for the sinusoidal aberrations in the previous section, the number of periods of the rectangular-pulse train was fixed at $N_{\text {samp }} / 2000$ (5 cycles per epoch) and the exponential decay set to $e^{-5 / N_{\text {samp }}}$. For rectangularpulse-train aberrations, the shorth method typically provided more accurate values for level(s) than did the histogram-mode method. However, us was typically smaller for one of

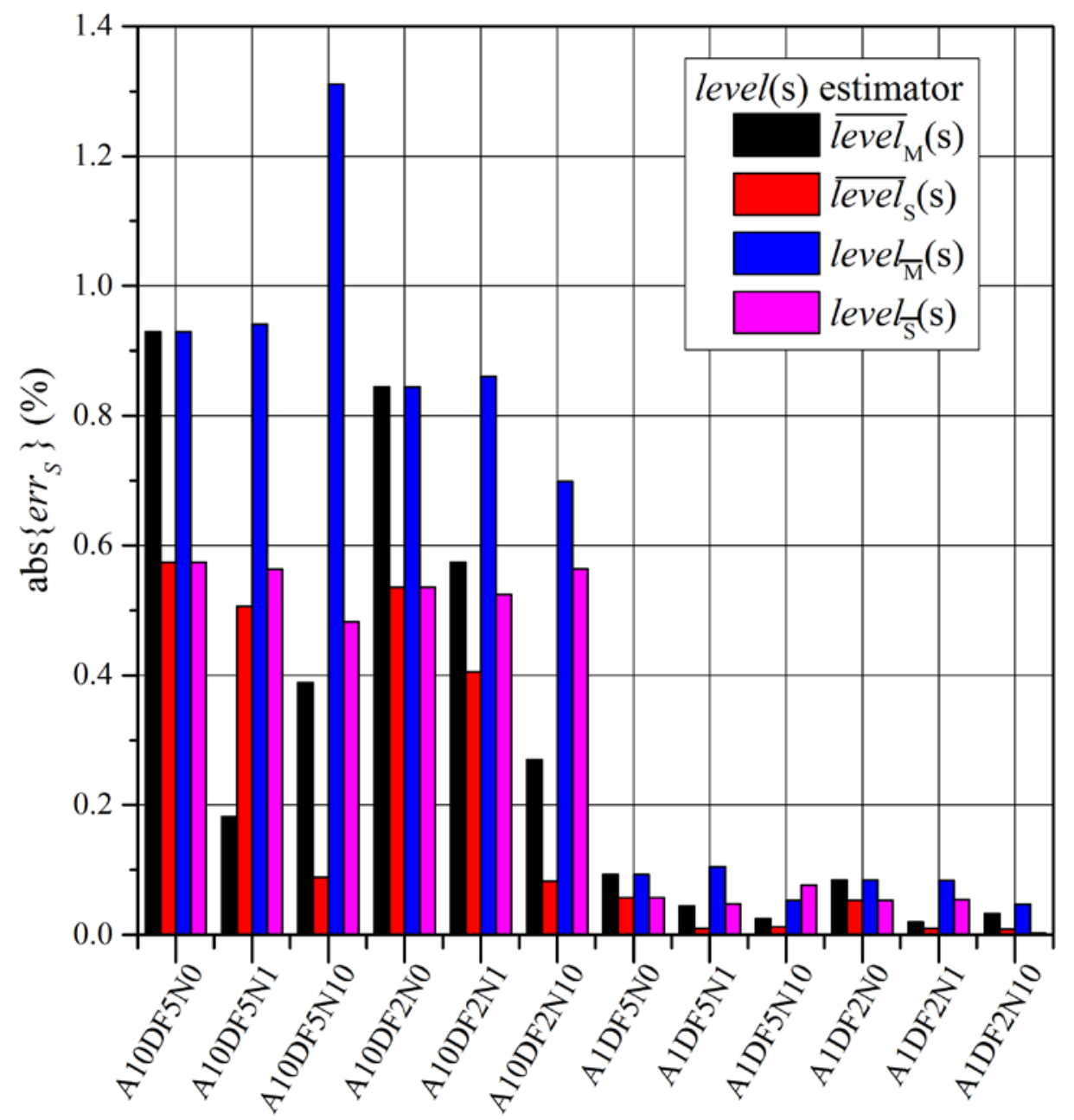

Fig. 11. Plot of abs $\{$ errs $\}$ for the different rectragular aberration

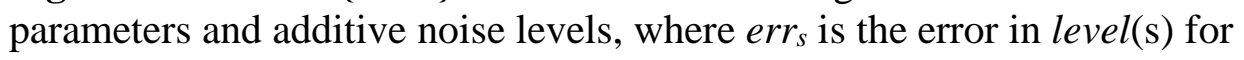

Horizontal annotation:

- A1, A10 - rectangular amplitude was set to $10 \%$ or $100 \%$ of level(Sref)

- DF2, DF5 - duty factor was set to20 \% or $50 \%$

- N0, N1, N10 - rms noise amplitude was set to $0 \%, 1 \%$, or 10 $\%$ of level(sref). 
the instantiations, $\overline{\text { level }}_{M}(\mathrm{~s})$, of the histogram-mode method than for either shorth method. The duty factor of the rectangular pulse train did not seem to affect the estimate of level(s) or $u_{s}$.

\subsection{Histogram-mode and shorth methods for noisy waveforms with impulse-like aberrations}

Figures 13 and 14 show the comparison for waveforms containing Chebyshev impulse-like aberrations plus additive noise. The peak amplitude of the aberration was 1.0 level( $\left.\mathrm{s}_{r e f}\right)$. The shorth method typically provided more accurate values for level(s) than did the histogrammode method for this set of waveforms. The cutoff frequency and filter order did not affect this observation. The simulations were repeated (data not shown) with the peak amplitude set to 0.1 level(sref). In that case, $\operatorname{err}_{S}$ and $u_{S}$ for level $\bar{M}_{\bar{M}}(\mathrm{~s})$ were both reduced to about onetenth of that shown in Figs. 13 and 14. For the other three level(s) estimators, the reduction

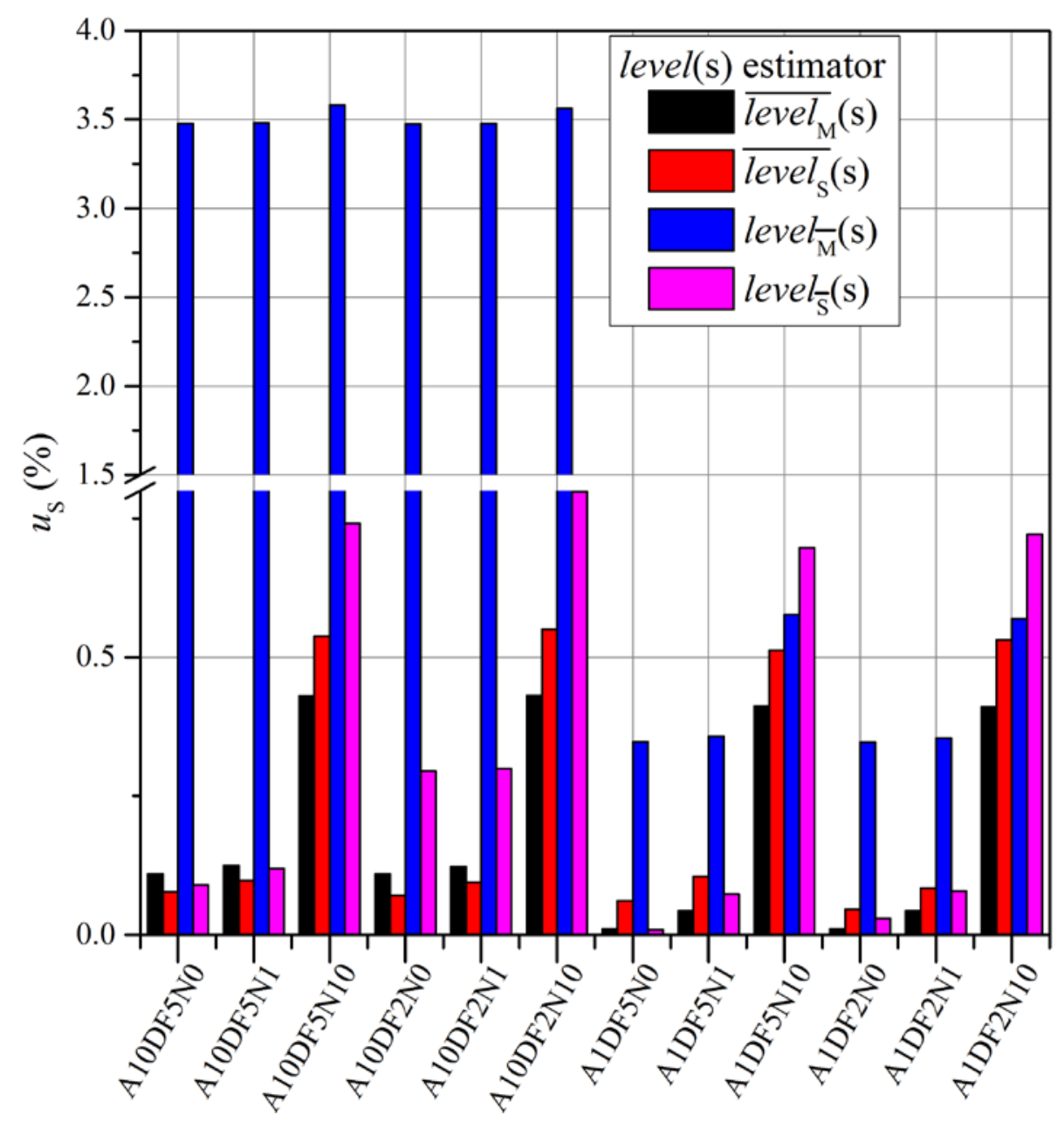

Fig. 12. The $u_{S}$ for the data shown in Fig. 11 . 
in $e r r_{S}$ and $u_{S}$ was dependent on the aberration and noise parameters but was not as significant as the reduction for level $_{\bar{M}}(\mathrm{~s})$.

\subsection{Collective comparison of histogram-mode and shorth methods}

Figures 5 through 14 show that the shorth method typically provides more accurate estimates of level(s) than does the histogram-mode method and that the measurement uncertainties are

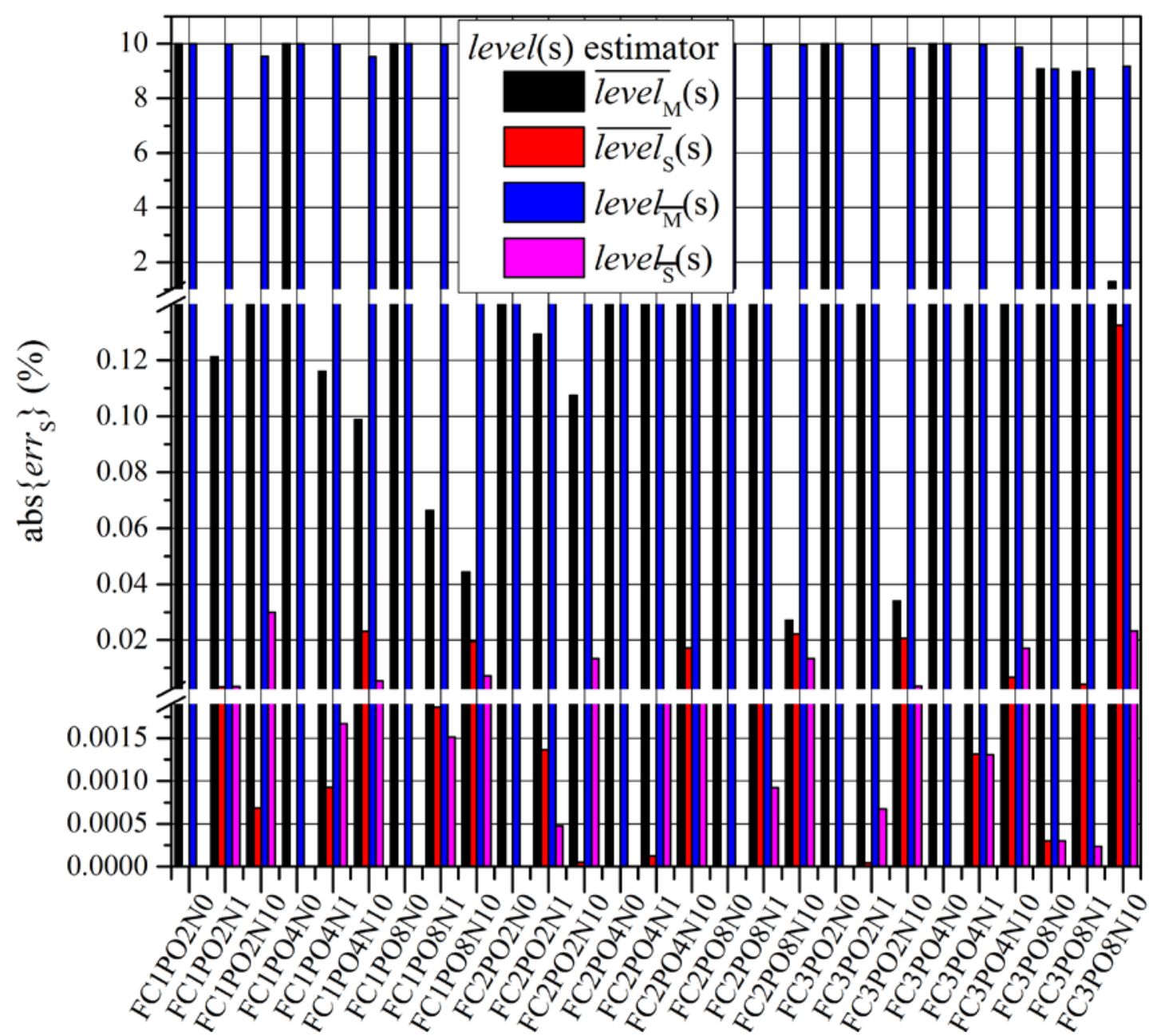

Fig. 13. Plot of abs\{errs $\}$ for the different impulse-like aberration parameters and additive noise levels, where $e r r_{s}$ is the error in level(s) for one of its four estimators (listed in the key) .

The impulse-like waveform was generated using a Chebyshev polynomial with adjustable parameters of cut-off frequency and order.

Horizontal annotation:

- FC1, FC2, FC3 - cut-off frequency was set to 0.1, 0.01, 0.0001 of the sampling frequency.

- PO2, PO4, PO8 - polynomial order was set to 2, 4, or 8

- N0, N1, N10 - rms noise amplitude was set to $0 \%, 1 \%$, or $10 \%$ of level( $\left(\mathrm{s}_{\mathrm{r} e f}\right)$. 
also typically less for the shorth method than for the histogram-mode method. The results in this section collectively summarize the observations shown in Figs. 5 through 14.

The number of different waveform types considered in this study is 84 , which each simulation being repeated 1000 times, resulting in a total of 84000 independent estimates of level(s) that are used to compare the level estimators, $\overline{\operatorname{level}}_{M}(\mathrm{~s}), \overline{\operatorname{level}}_{s}(\mathrm{~s})$, level $_{\bar{M}}(\mathrm{~s})$, and level $_{\bar{S}}(\mathrm{~s})$. Figures 15 and 16 show the distributions of err $_{\mathrm{s}}$ and $u_{\mathrm{s}}$ for the different level estimators. These distributions, although sparse because of the small number (84) of waveform aggregates, are nominally similar to those given in [9], where the distributions in [9] are the result of 4000 individual waveforms. The distributions for either the histogrammode method or the shorth method shown in Figs. 15 and 16 have a greater range when using one averaged waveform ( level $_{\bar{M}}(\mathrm{~s})$ and level $_{\bar{S}}(\mathrm{~s})$ ) than when using the average of a set of waveforms $\left(\overline{\text { level }}_{M}(\mathrm{~s})\right.$ and $\left.\overline{\text { level }}_{S}(\mathrm{~s})\right)$. This observation is consistent with [9]. The randomappearing results for level $_{\bar{M}}(\mathrm{~s})$ shown in Figs. 15 and 16 are a result of the very broad distribution, the sparse number of samples (84 waveform aggregates), and keeping the range

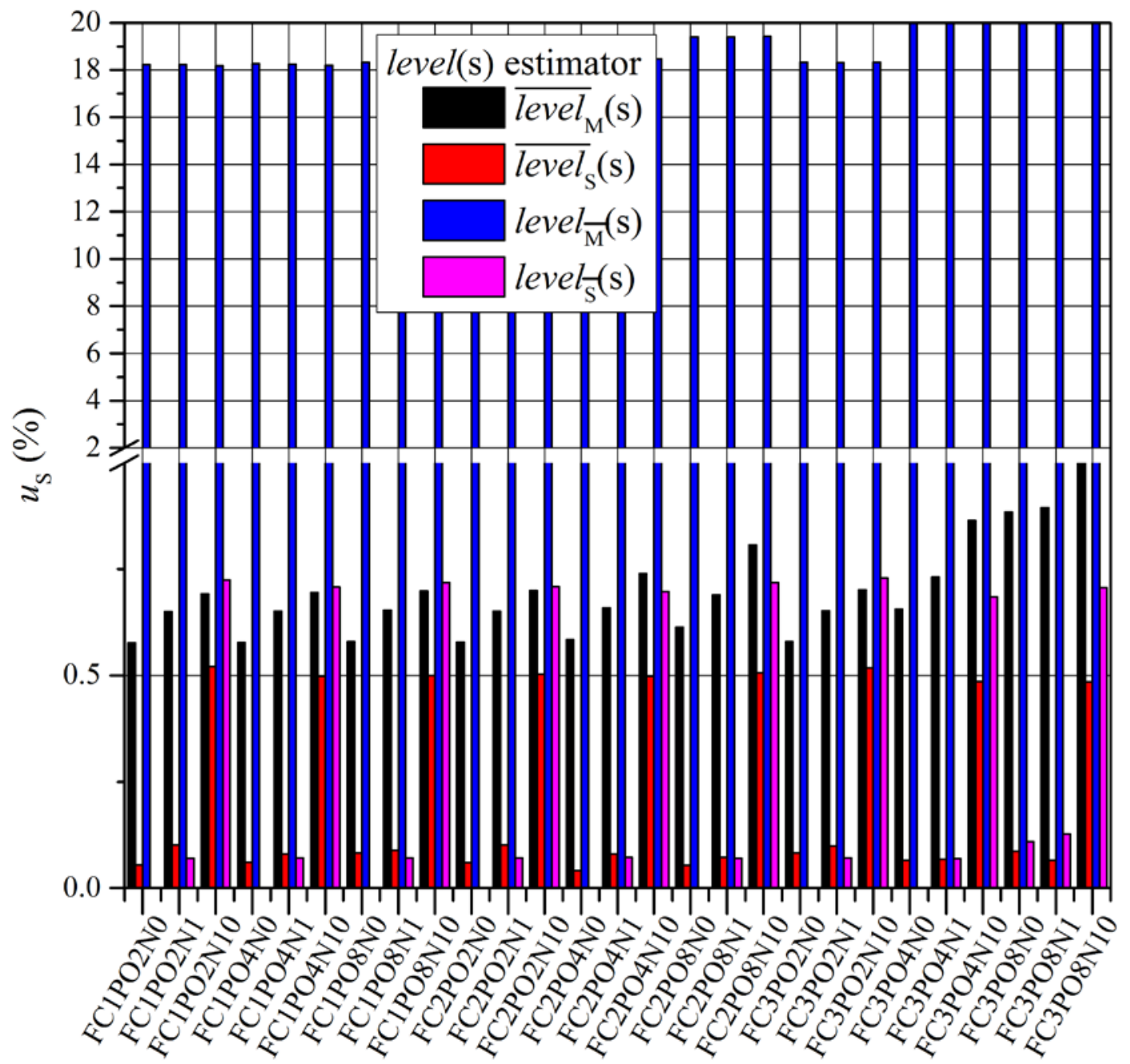

Fig. 14. The $u_{s}$ for the data shown in Fig. 13. 
of the horizontal axis of the plots narrow so that the structure of the distributions for the other level estimators can be readily observed. The statistics of these distributions for all of the simulations performed are summarized in Table 3.
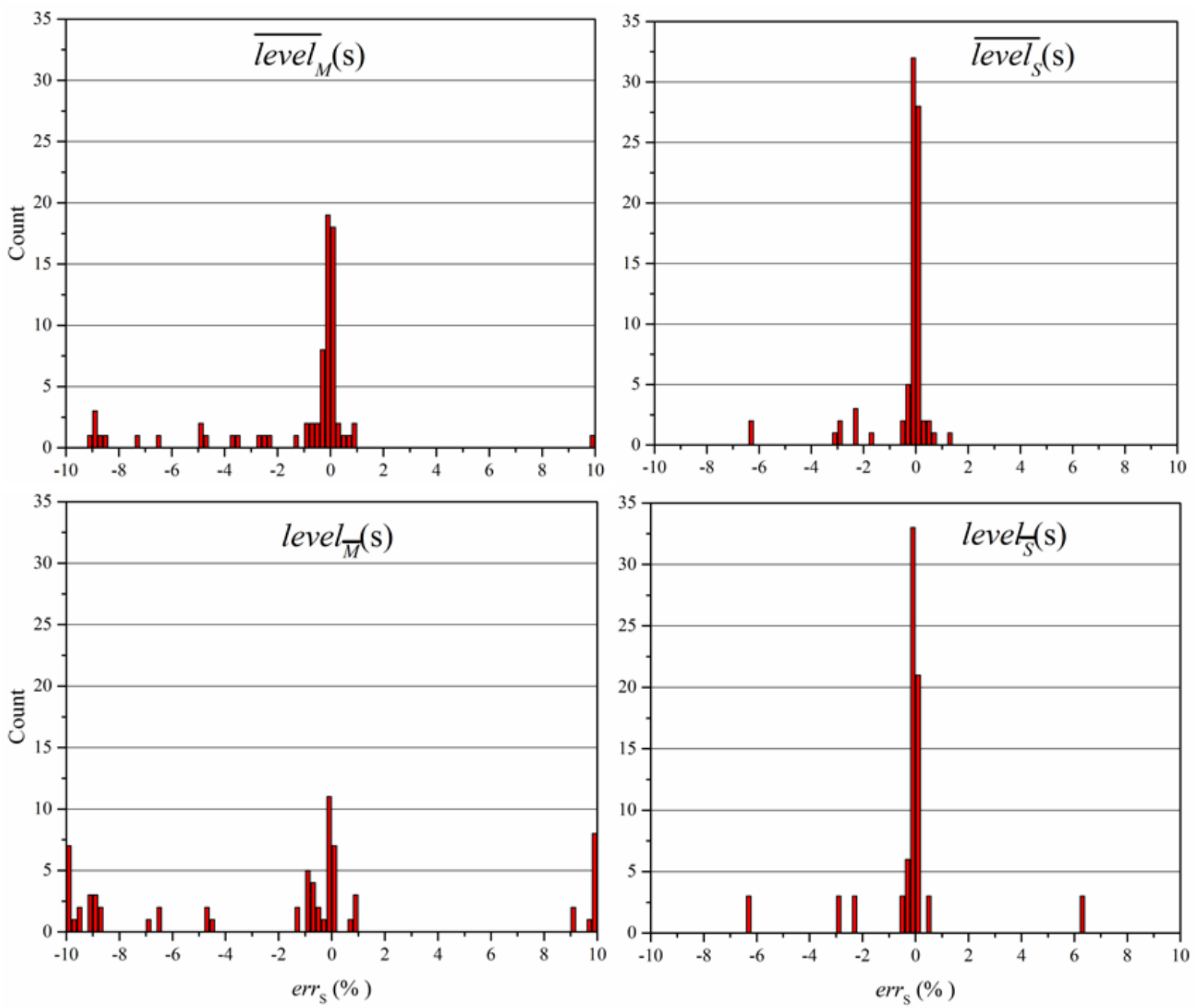

Fig. 15. Distribution of errs for the different level estimators, where errs is given as a

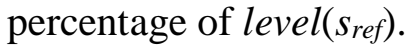

The horizontal range of the plots was fixed to a common range to facilitate comparison of the plots but which resulted in truncating the data for some of the distributions. 

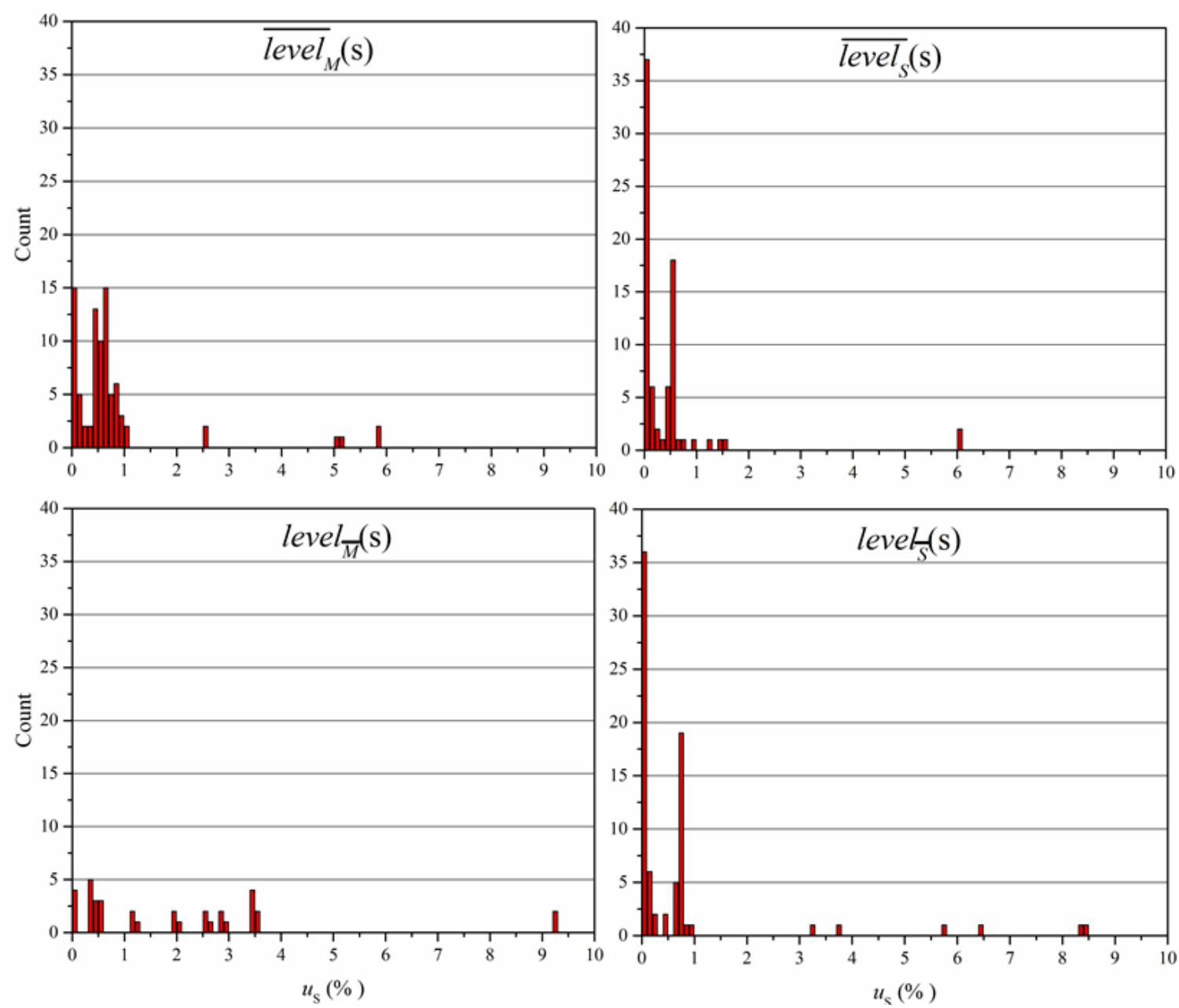

Fig. 16. Distribution of $u_{s}$ for the different level estimators, where $u_{s}$ is given as a

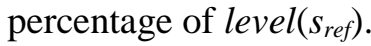

The horizontal range of the plots was fixed to a common range to facilitate comparison of the plots but which resulted in truncating the data for some of the distributions.

The shorth method typically provided lower $a b s\left\{e r r_{s}\right\}$ and $u_{s}$ than did the histogram-mode method and this may be due the inherent nature of the shorth method, which discards a significant portion of $N_{\text {samp }}$ before taking the mean of the retained values. This reduction in $N_{\text {samp }}$ results in a reduced range of waveform values for the shorth method. This range reduction, from a measurement-uncertainty perspective, is advantageous for the shorth method when compared to the histogram mode method because the magnitude of many of the histogram-mode and shorth method uncertainty contributors, such as, $u_{b i n}, u_{0}, \sigma_{n}, \sigma_{M}, u_{c o v}$, and $\sigma_{S}$, increase with an increasing range of waveform values. Similarly, the $a b s\left\{e r r_{s}\right\}$ would be expected to be less for the shorth method than for the histogram-mode method for the same reason. 
Table 3. Errors in the estimates of level(s) and their combined measurement uncertainties for the given estimators of level(s) for all of the different noise and aberration simulations performed. All values are given as percentage of level(s).

\begin{tabular}{|c|c|c|c|c|}
\hline & Mean & $\begin{array}{c}\text { Standard } \\
\text { Deviation }\end{array}$ & Maximum & Minimum \\
\hline$\overline{\text { level }}_{M}(\mathrm{~s})-$ level $\left(\mathrm{s}_{\text {ref }}\right)$ & $-2.39 \mathrm{E}+00$ & $1.44 \mathrm{E}+01$ & $1.00 \mathrm{E}-01$ & $-9.00 \mathrm{E}-01$ \\
\hline$\overline{\text { level }}_{s}(\mathrm{~s})-$ level $\left(\mathrm{s}_{\text {ref }}\right)$ & $-3.58 \mathrm{E}-01$ & $9.95 \mathrm{E}+00$ & $6.36 \mathrm{E}-01$ & $-6.36 \mathrm{E}-01$ \\
\hline level $_{\bar{M}}(\mathrm{~s})-$ level $\left(\mathrm{s}_{\text {ref }}\right)$ & $-4.71 \mathrm{E}+00$ & $2.47 \mathrm{E}+01$ & $9.09 \mathrm{E}-01$ & $-9.08 \mathrm{E}-01$ \\
\hline level $_{\bar{S}}(\mathrm{~s})-$ level $\left(\mathrm{s}_{\text {ref }}\right)$ & $-1.74 \mathrm{E}+00$ & $1.70 \mathrm{E}+01$ & $6.36 \mathrm{E}-01$ & $-6.37 \mathrm{E}-01$ \\
\hline$u_{M, W, 2}$ & $7.67 \mathrm{E}-01$ & $1.14 \mathrm{E}+00$ & $5.82 \mathrm{E}-02$ & $0.00 \mathrm{E}+00$ \\
\hline$u_{S, W, 2}$ & $3.96 \mathrm{E}+00$ & $1.38 \mathrm{E}+01$ & $6.68 \mathrm{E}-01$ & $0.00 \mathrm{E}+00$ \\
\hline$u_{M, \bar{W}, 2}$ & $1.89 \mathrm{E}+01$ & $2.84 \mathrm{E}+01$ & $1.37 \mathrm{E}+00$ & $0.00 \mathrm{E}+00$ \\
\hline$u_{S, \bar{W}, 2}$ & $6.13 \mathrm{E}+00$ & $1.98 \mathrm{E}+01$ & $9.06 \mathrm{E}-01$ & $0.00 \mathrm{E}+00$ \\
\hline
\end{tabular}

\subsection{Effects of $N_{w v f m}$ and $N_{s a m p}$ on the $e r r_{s}$ and $u_{S}$}

The effects of $N_{w v f m}$ and $N_{\text {samp }}$ on the errs and $u_{S}$ are examined here. As would be expected, increasing $N_{\text {wvfm }}$ or $N_{\text {samp }}$ decreases errs and $u_{\text {s. }}$ Figure 17 corroborates the data shown in Fig. 5 and shows that the histogram-mode method produces greater errs than does the shorth method. Furthermore, the estimators, $\operatorname{level}_{\bar{M}}(\mathrm{~s})$ and $\operatorname{level}_{\bar{S}}(\mathrm{~s})$, that use the data of $\bar{W}[t]$ alone produce smaller errs than the estimators, $\overline{\text { level }}_{M}(\mathrm{~s})$ and $\overline{\text { level }}_{S}(\mathrm{~s})$, that use the data from all of the $W_{i}[t]$ that contribute to $\bar{W}[t]$. The data in Fig. 18 supports the observation (see Fig. 6) that histogram-mode method produces smaller $u_{\text {s }}$ than does the shorth method and, that, typically the instantiations of the histogram-mode method or shorth method that uses information from the $W_{i}[t]$ produces smaller uncertainties than do the instantiations that use the $\bar{W}[t]$ alone. This data also show that $N_{\text {wvfm }}$ should be greater than 1000 and $N_{\text {samp }}$ should be greater than 100 to minimize errs and $u_{S}$. It is expected that variation in $N_{w v f m}$ and $N_{\text {samp }}$ will affect errs and $u_{S}$ similar to the situation with only additive noise, as shown in Figs. 17 and 18. This is because the histogram-mode and shorth methods are based on the distribution of values, and with fewer values (small $N_{\text {samp}}$ ) for the same aberration amplitude range, the distributions will be more coarse than for large $N_{\text {samp }}$. Large $N_{\text {wvfm }}$ should then be able to compensate for small $N_{\text {samp }}$ due to averaging, and this is shown in Figs. 17 and 18. 


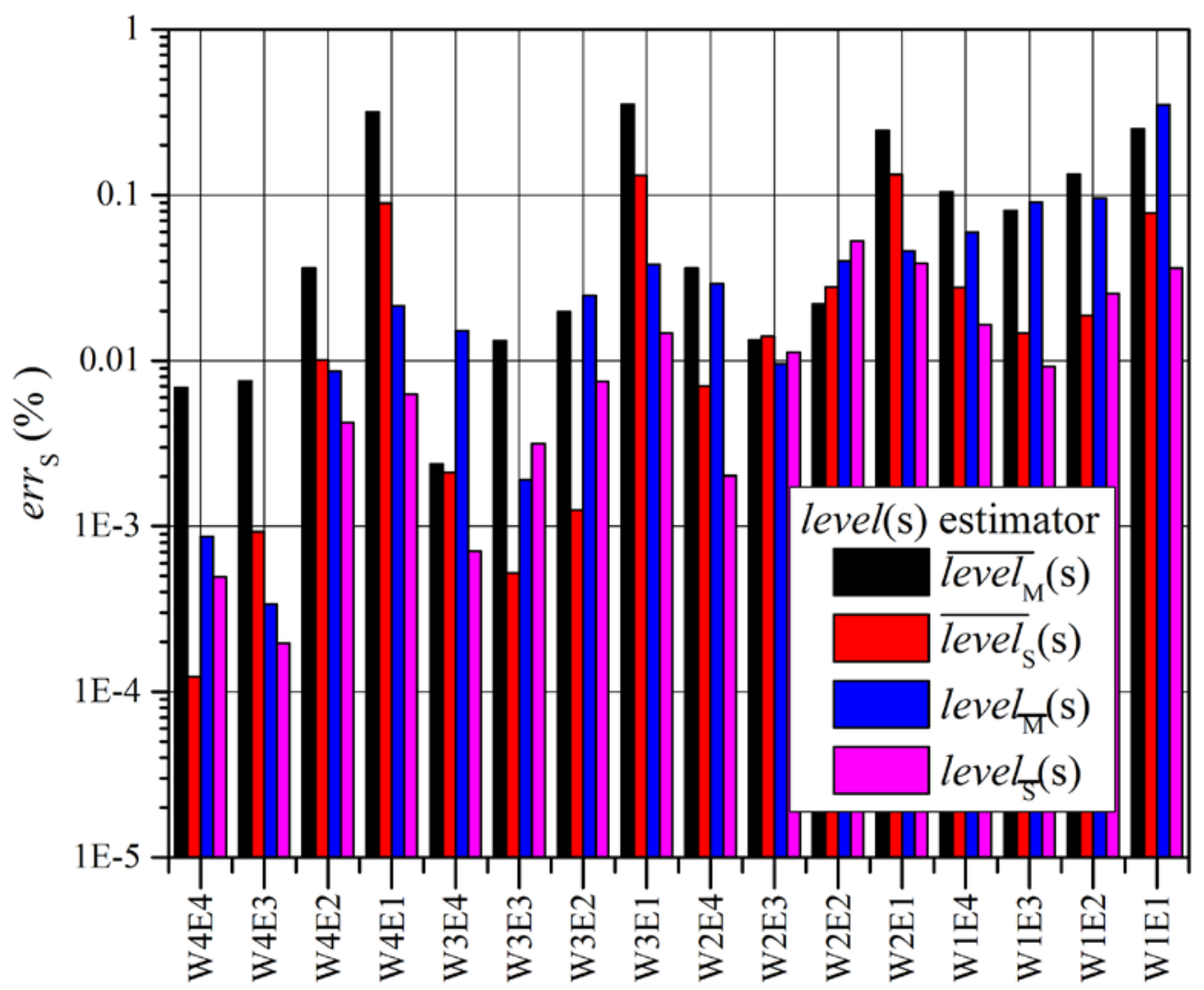

Fig. 17. Plot of abs $\left\{e r r_{S}\right\}$ for the different additive noise levels, different number of elements/samples in a waveform, and different number of waveforms averaged for the four estimators of level(s) (listed in the key) .

Horizontal annotation:

- W4, W3, W2, W1 - $N_{\text {wrfm }}$ was set to $10000,1000,100$, or 10

- E4, E3, E2, E1 - $N_{\text {samp }}$ was to $10000,1000,100$, or 10

- N0, N1, N10 - rms noise amplitude was set to $0 \%, 1 \%$, or $10 \%$ of level( $\left.\mathrm{s}_{\text {ref }}\right)$.

\section{Conclusions}

The histogram-mode method is the most commonly-used method for estimating the state levels of a waveform. The shorth method, initially developed to approximate the mode, was introduced in 2009 as another method for computing the state levels. The effect of both methods on the accuracy and measurement uncertainty of the estimate of the state level of the waveform was examined. The shorth method typically provides lower uncertainty values for the state level than does the histogram-mode method for the aberrations and noise levels examined here. However, the difference in state-level uncertainty between the histogrammode method and the shorth method for typical aberration levels and noise levels is at least 10 times less than the amplitude noise found in waveforms of typical signals. Consequently, either the histogram-mode method or shorth method could be used without a noticeable difference in a reported measurement uncertainty for state level. The instantiations of either the histogram-mode or shorth methods that use only information on the average waveform and its histogram produce larger errors and uncertainties in the value of state level than if 
information on all contributing waveforms and their histograms are used. Accordingly, only the latter instantiations of the methods should be used.

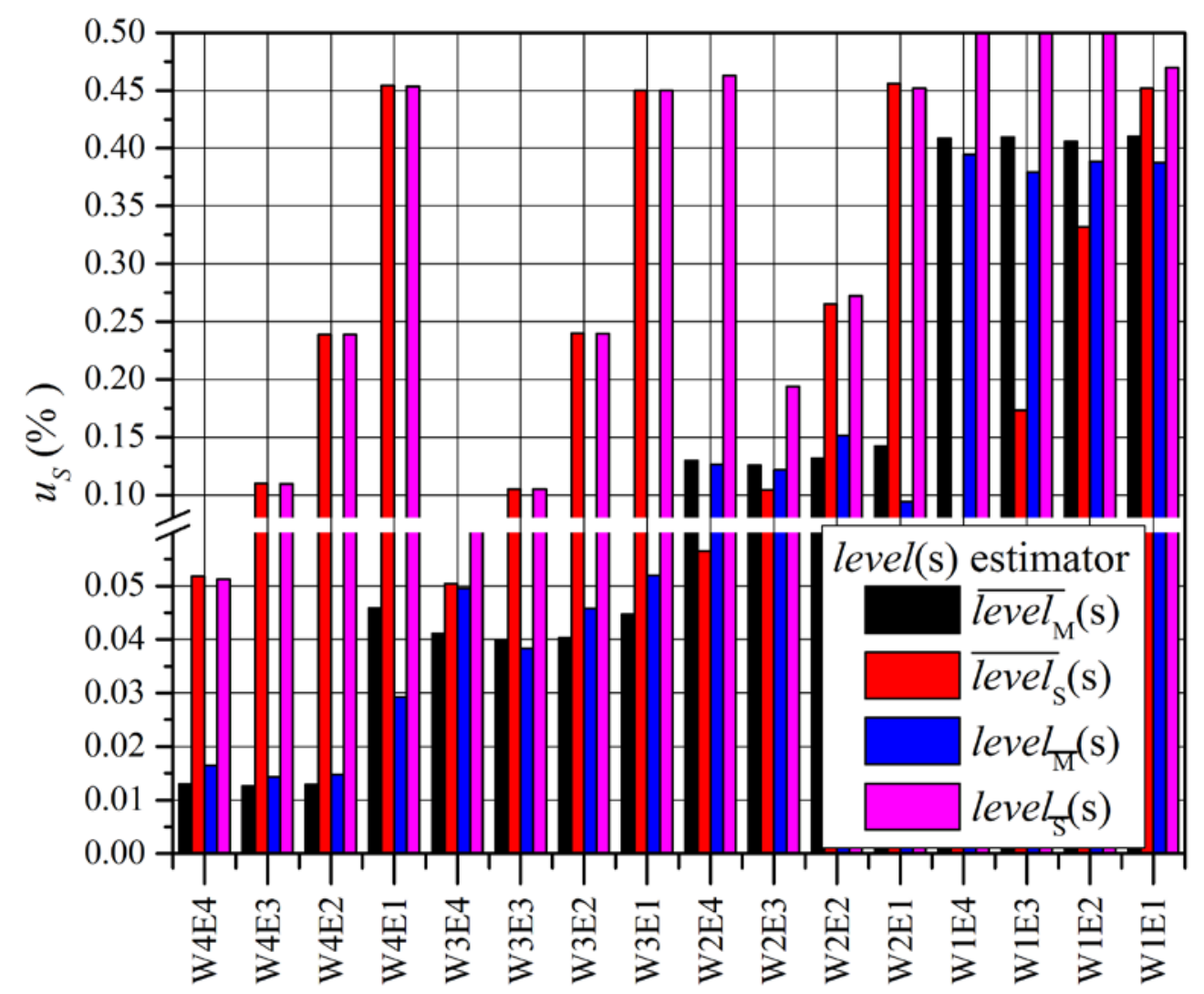

Fig. 18. The uncertainty in level(s) for the data shown in Fig. 17. 


\section{References}

[1] International Electrotechnical Commission (2013) IEC 60469, Edition 1.0 2013-04, Transitions, pulses and related waveforms - Terms, definitions and algorithms (International Electrotechnical Commission, Geneva, Switzerland), 1st Ed.

[2] Institute of Electrical and Electronic Engineers (2011) IEEE Std. 181-2011, IEEE Standard for Transitions, Pulses, and Related Waveforms (Institute of Electrical and Electronics Engineers, New York, USA).

[3] International Electrotechnical Commission (2017) IEC 62754, Edition 1.0 2017-05, Computation of waveform parameter uncertainties (International Electrotechnical Commission, Geneva, Switzerland), 1st Ed.

[4] Paulter NG and Larson DR (2002) Pulse parameter uncertainty analysis. Metrologia 39: 143 to 155. https://doi.org/10.1088/0026-1394/39/2/4

[5] Paulter NG (1998) The Effect of Histogram Size on Histogram-Derived Pulse Parameters. IEEE Transactions on Instrumentation and Measurement 47(3): 609-612. https://doi.org/10.1109/19.744309

[6] Solomon OM, Larson DR, and Paulter NG (2001) "Comparison of some algorithms to estimate the low and high state level of pulses. IEEE Instrumentation and Measurement Technology Conference, Budapest, Hungary, pp. 96-101. https://doi.org/10.1109/IMTC.2001.928794

[7] Andrews DF, Bickel PJ, Hampel FR, Huber PJ, Rogers WH, and Tukey JW (1972) Robust Estimates of Location, (Princeton University Press, Princeton, NJ).

[8] Hale PD and Wang CM (2009) Calculation of pulse parameters and propagation of uncertainty. IEEE Transactions on Instrumentation and Measurement 58(3): 639648. https://doi.org/10.1109/TIM.2008.2005560

[9] Bieler M and Paulter NG (2016) Estimation of waveform state levels and uncertainties using the histogram and shorth methods, Conference on Precision Electromagnetic Measurements, CPEM 2016, pp. 10-15. https://doi.org/10.1109/CPEM.2016.7540806

[10] Paulter NG, Smith AJA, Larson DR, Souders TM, and Roddie AG (2003) NISTNPL interlaboratory pulse measurement comparison. IEEE Transactions on Instrumentation and Measurement 52: 1825-1833.

https://doi.org/10.1109/TIM.2003.820456

[11] Paulter NG and Larson DR (2002) NIST's High-speed Pulse Parameter Measurement Service IEEE Instrumentation and Measurement Technology Conference, Anchorage, AK, USA, pp. 625-628. https://doi.org/10.1109/IMTC.2002.1006914 
Annex A. Method for computing histogram mode values (taken with modification from $[1,2])$

a) Determine the maximum and minimum amplitude values, $y_{\max }$ and $y_{\min }$ of $W[t]$ by searching $\mathrm{W}[\mathrm{t}]$ for $y_{\min }$ and $y_{\max }$.

b) Calculate the amplitude range, $y_{R}$, of $W[t]$ using: $y_{R}=y_{\max }-y_{\min }$.

c) Calculate the bin width, $\Delta y$, using:

$$
\Delta y=\frac{y_{R}}{N_{b i n}}=\frac{y_{\max }-y_{\min }}{N_{b i n}} .
$$

d) Initially set $B_{j}=0$ for $j=1 \ldots J$, where $B_{j}$ is the count for the $j^{\text {th }}$ histogram bin. Sort through the values, $y_{i}, i=1, N$, where $N$ is the number of values of $W[t], N_{b i n}$ is the number of bins for a given histogram, and if $y_{i}$ lies within the range of a bin, that is if:

$$
\left(y_{\min }+(j-1) \Delta y\right) \leq y_{i}<\left(y_{\min }+j \Delta y\right)
$$

for $1 \leq j \leq N_{b i n}$, then set $B_{j}=B_{j}+1$.

e) Identify the $B_{i}$ with the largest number of counts. This is the mode. 
Annex B. Method for computing shorth values (taken with modification from 1,2)

The method described here starts after identification of the state occurrence. Information on how to segregate the waveform is found in $[1,2]$.

a) Reorder the waveform values of the state occurrence, (s), into a non-decreasing sequence to give

$$
(\mathrm{s})_{n d}=y_{(1)} \leq y_{(2)} \leq \ldots \leq y_{\left(N_{S}\right)},
$$

where $N_{S}$ is the number of samples in the shorth interval and (s) indicates a state occurrence. For clarity, $y_{i}$ is not necessarily equal to $y_{(i)}$.

b) Perform the following to compute the shorth collection for (s) $n d$ :

$$
h=\left\lfloor f_{s} N_{\text {samp }}\right\rfloor+1,
$$

where $f_{s}$ is the fraction of $N_{\text {samp }}$ used in the shorth collection, which was set to 0.5 for the simulations performed in this work, $\lfloor x\rfloor$ is the greatest integer less than or equal to $x$

min_diff $=10^{9}$

$$
d=N_{\mathrm{S}}-h+1
$$

for $i=1 . . d$

$$
\begin{gathered}
\text { diff }=y_{(h+i-1)}-y_{(i)} \\
\text { if }(\text { diff }<\text { min_diff }) \text { then } \\
\text { min_diff }=\text { diff } \\
m=i
\end{gathered}
$$

endif

endfor

c) Set the shorth collection, $S_{\mathrm{c}}$, for (s):

$$
S_{\mathrm{c}}=\left(y_{(m)}, \ldots, y_{(h+m-1)}\right) \text {. }
$$

Note: The algorithm above produces a shorth collection that, if two or more successive intervals qualify for the shorth, selects the first interval.

d) The level(s) is computed using:

$$
\operatorname{level}(s)=\frac{1}{h} \sum_{j=m}^{h+m-1} y_{(j)} \text {. }
$$




\section{Annex C. Aberration generating functions}

- Impulse-like, $F_{1}$ :

$$
F_{1}=\frac{1}{\sqrt{1+\varepsilon^{2} T_{n}^{2}\left(\frac{1}{f_{c}}\right)}},
$$

where $f_{c}$ is the cutoff frequency, the values of which are given in Table $1 ; T_{n}$ is the Chebyshev polynomial of order $n$, the values of which are given in Table 1 ; and $\varepsilon$ is the ripple.

- Sinusoidal, $F_{2}$ :

$$
F_{2}=A \sin \left(\frac{2 \pi i}{T}\right)
$$

where $i=1, \ldots, N$; $A$ is the amplitude and $T$ is the period, the values of both are given in Table 1.

- Decaying sinusoidal, $F_{3}$ :

$$
F_{3}=F_{2} e^{-x / i}
$$

where $i=1, \ldots, N$; the values of $x$ are given in Table 1 .

- Rectangular, $F_{4}$ :

$$
\begin{aligned}
& F_{4}=\sum_{i=1}^{5} u\left[t_{0, i}, t_{f, i}\right] \\
& u\left[t_{0, i}, t_{f, i}\right]==\begin{array}{c}
1, \text { for } t_{0, i} \leq \mathrm{t} \leq \mathrm{t}_{f, i} \\
0, \text { otherwise }
\end{array} \\
& t_{0, i}=(1-d f) 2000+t_{f, i-1} \\
& t_{f, i}=2000 i, i=0,1, \ldots, 5
\end{aligned}
$$

- Decaying rectangular, $F_{5}$ :

$$
F_{5}=F_{4} e^{-5 / i}, i=1, \ldots, N
$$

